\title{
Los jóvenes ninis en el Ecuador
}

Recibido: noviembre, 23 de 2017 - Aprobado: marzo, 22 de 2018

Doi: http:/ /dx.doi.org/10.12804/revistas.urosario.edu.co/economia/a.6800

\author{
Karina Buitrón* \\ Verónica Jami ${ }^{\dagger}$ \\ Yasmín Salazar Méndez $\ddagger$
}

\section{Resumen}

Los "ninis" hacen referencia al grupo de jóvenes entre 15 y 24 años que ni estudian ni trabajan. Factores individuales, como el ingreso familiar y la educación, incidirían en la formación de los jóvenes ninis. En este artículo se analizan las características de los jóvenes ninis ecuatorianos y se determinan los factores que aumentan la probabilidad de que un joven no estudie ni trabaje. Para esto, modelos logit son estimados a partir de la Encuesta de Condiciones de Vida del 2014. Los resultados sugieren que los jóvenes de familias de baja renta, las mujeres, los jóvenes que habitan en el área urbana y los pertenecientes a las minorías étnicas son más propensos a convertirse en un nini.

Palabras clave: educación y desigualdad, capital humano, bienestar.

Clasificación JEL: I24, I31, J24

* Carrera de Ingeniería en Ciencias Económicas y Financieras de la Escuela Politécnica Nacional.

+ Carrera de Ingeniería en Ciencias Económicas y Financieras de la Escuela Politécnica Nacional.

‡ Departamento de Economía Cuantitativa de la Escuela Politécnica Nacional. Correspondencia: Ladrón de Guevara E11.253 [PO·Box 17-01-2759], Quito, Ecuador. Correo electrónico: yasmin.salazar@epn.edu.ec

Cómo citar este artículo: Buitrón, K., Jami, V. \& Salazar Méndez, Y. (2018). Los jóvenes ninis en el Ecuador. Revista de Economía del Rosario, 21(1), 39-80.

Doi: http://dx.doi.org/10.12804/revistas.urosario.edu.co/economia/a.6800 


\title{
NEETs in Ecuador
}

\begin{abstract}
The NEETs refers to young people between 15 and 24 who are neither in employment nor in education or training. Individual factors, such as family income and education, could influence the appearence of the NEETs. This article analyzes the characteristics of the Ecuadorian NEETS and determines the factors that increase the probability that a young person is neither in employment nor in education or training. For this purpose, Logit models based on the national Living Conditions Survey (2014) are estimated. The results suggest that young people from low-income families, women, urban youth and those belonging to ethnic minorities are more likely to become a NEET young.
\end{abstract}

Keywords: education and inequality, human capital, welfare. JEL Classification: I24, I31, J24

\section{Os Jovens Nem-nem no Equador}

\section{Resumo}

Os 'nem-nem' fazem referência ao grupo de jovens entre 15 e 24 anos que nem estuda nem trabalha. Fatores individuais, como o ingresso familiar e a educação incidiriam na formação dos jovens nem-nem. Neste artigo se analisam as características dos jovens nemnem equatorianos e se determinam os fatores que aumentam a probabilidade de que um jovem não estude nem trabalhe. Para isto, modelos logit são estimados a partir do Inquérito de Condições de Vida (2014). Os resultados sugerem que os jovens de famílias de baixa renda, as mulheres, os jovens que habitam na área urbana e os pertencentes às minorias étnicas são mais propensos a se tornar em um nem-nem.

Palavras-chave: educação e desigualdade, capital humano, bem-estar.

Classificação JEL: I24, I31, J24 


\section{Introducción}

En el mundo existen 260 millones de ninis, es decir, de jóvenes entre 15 y 24 años que ni estudian ni trabajan (De Hoyos, Rogers \& Székely, 2016). Esta cifra, que equivale al 22,4\% de la población joven, ha encendido las alarmas de los gobiernos de diversos países y de organismos internacionales, como la Organización de las Naciones Unidas, que en el 2015 incluyó en la Agenda 2030 para el Desarrollo Sostenible un objetivo relacionado con la reducción de los jóvenes ninis. ${ }^{1}$

La atención que el grupo nini ha generado en los últimos años no solo está ligado al hecho de que es una "cifra" que ha ido aumentando con el tiempo, pues las consecuencias que la inactividad de los jóvenes acarrea tanto a nivel agregado como individual, a corto y largo plazos obligan a tomar correctivos urgentes. Hanushek y Woessmann (2008) argumentan que la productividad y el crecimiento económico a largo plazo dependen de la calidad del capital humano de una sociedad, y que este es un aspecto indispensable para impulsar la innovación y adaptar nuevas tecnologías. Como el grupo de ninis está compuesto por jóvenes que no realizan ninguna actividad, este sector representa un capital humano desperdiciado e improductivo, y, por tanto, las oportunidades de crecimiento económico de un país se verían reducidas.

Además, los jóvenes ninis traen consigo varios riesgos, como la contribución a la transmisión intergeneracional de la desigualdad. En el caso latinoamericano casi el $60 \%$ de los ninis proviene de hogares pobres o vulnerables y que están localizados en los cuatro deciles más bajos de la distribución del ingreso. Este hecho podría aumentar las desigualdades existentes, obstruyendo tanto la movilidad social y económica, como la reducción de la pobreza a largo plazo (Vakis, Rigolini \& Lucchetti, 2015; Ferreira, Messina, Rigolini, López-Calva \& Vakis, 2013).

A nivel individual, según la Comisión Económica para América Latina y el Caribe (Cepal, 2014), los jóvenes ninis llegan a su vida adulta con pocas oportunidades de trabajo, educación o formación, generando efectos adversos a nivel individual, como problemas emocionales, sociales y psicológicos, pero también en toda la sociedad, debido a que constituyen una fuerza de trabajo improductiva que no genera capital a través de un sistema formal

1 “Objetivo 8. Promover el crecimiento económico sostenido, inclusivo y sostenible, el empleo pleno y productivo y el trabajo decente para todos. Objetivos específicos: 8.5 De aquí a 2030, lograr el empleo pleno y productivo y el trabajo decente para todas las mujeres y los hombres, incluidos los jóvenes y las personas con discapacidad, así como la igualdad de remuneración por trabajo de igual valor. 8.6 De aquí a 2020, reducir considerablemente la proporción de jóvenes que no están empleados y no cursan estudios ni reciben capacitación" (ONU, 2015). 
de trabajo, ocasionando mayor pobreza, un aumento en la brecha económica entre las generaciones jóvenes y adultas, y exclusión social.

Las consecuencias a las que se enfrentarán los ninis pueden presentarse en el corto y largo plazos, pues la condición de ser nini hoy conducirá a efectos negativos dentro de veinte años; es decir, cuando el joven atraviese sus mayores años de productividad será más propenso a tener menores ingresos. El Banco Mundial (Hoyos et al., 2016) señala que, en el caso de los hombres, el ser nini está asociado con una disminución de $7 \%$ en los ingresos individuales dentro de veinte años y, para el caso de las mujeres esta reducción sería del 3\%.

En América Latina, el tema nini también ha cobrado importancia debido a su tendencia creciente. Según el Banco Mundial (Hoyos et al., 2016), de los 110 millones de jóvenes con edades entre 15 y 24 años, aproximadamente 20 millones, que corresponden al 20,3 \% del total de la población de jóvenes, son considerados ninis; es decir, 1 de cada 5 jóvenes en la región ni estudian, ni trabajan. Por otro lado, en un estudio publicado por la Corporación Andina de Fomento - $\mathrm{CAF}{ }^{2}$ (Berniell et al., 2016) se indica que el número de jóvenes ninis entre 15 y 25 años se ha mantenido estable en los últimos veinte años (19 millones) y que, inclusive, ha existido una reducción del $23 \%$ al $19 \%$ de ninis, en el periodo 1992-2014. La CAF también señala que los países con menor presencia nini son Bolivia (13\%) y Perú $(14,1 \%),{ }^{3}$ mientras que Honduras y Guatemala muestran los mayores porcentajes: $26,8 \%$ y $27,7 \%$, respectivamente. A pesar de que, según la misma fuente, en general se observa una disminución en el porcentaje de ninis, Argentina, Bolivia y Ecuador llaman la atención por mostrar un incremento entre 1992 y el 2014. Ecuador es el país que muestra el mayor crecimiento de ninis ( $2 \%$ y con el fin de contrarrestar el creciente número de jóvenes desempleados, entró en vigencia desde el 2016 la Ley Orgánica para la Promoción del Trabajo Juvenil. En esta se plantea que las empresas deben contratar a jóvenes entre los 18 y 26 años que ingresan por primera vez al empleo adecuado, quienes recibirán como mínimo una remuneración de un salario básico unificado. Sin embargo, la contratación de este grupo no debe superar el $20 \%$ del total de la nómina.

A pesar del interés en el tema nini de algunos organismos (como el Banco Mundial, la Cepal, la CAF, la ONU), este ha sido abordado, en su mayoría, a nivel regional. La información disponible hasta el momento constituye, sin duda, un importante punto de partida para prestar atención a los jóvenes ninis

2 La CAF indica que sus conclusiones fueron obtenidas del estudio de Tornarolli (2016) y que las diferencias pueden deberse a las diversas metodologías.

3 La presencia de jóvenes en el área rural incidiría en esto, pues a pesar de que ellos no estudien deben trabajar. 
y proponer soluciones, sin embargo, el conocimiento de las particularidades de los ninis de cada país puede facilitar la concepción de políticas específicas, acorde con las condiciones y necesidades de cada territorio.

Así, este artículo tiene como objetivo caracterizar al grupo de jóvenes ninis del Ecuador. Para esto, son estimados modelos de variable dependiente binaria utilizando la Encuesta de Condiciones de Vida (2014). El artículo está organizado de la siguiente manera. Además de esta sección introductoria, en la sección 2 se presenta un apartado que muestra la importancia de qué los jóvenes se preparen adecuadamente. La siguiente sección incluye información general sobre los jóvenes ninis, los orígenes del término, lo que se conoce sobre los factores que inciden en la formación de este grupo y las políticas que se pueden implementar para hacer frente al problema. En la sección 4 se muestran los datos y la metodología, y se hace un análisis de estadística descriptiva de los ninis ecuatorianos. En la sección 5 se presentan los resultados de la estimación y, finalmente, en la sección 6 se presenta las conclusiones del artículo.

\section{1. ¿Por qué es importante que los jóvenes estudien?}

El reconocimiento de la importancia de la educación, tanto para el desarrollo individual como para el de la sociedad en general, no es nuevo. Smith (1952) mencionaba la importancia de desarrollar habilidades y destrezas para realizar un trabajo y, de esta manera, incrementar la productividad. Más tarde, Mincer (1958) indicaba que la capacitación o el aprendizaje tienen un efecto positivo tanto en el trabajo como en el aumento de los ingresos, es decir, en el futuro las personas tendrán mayor probabilidad de aumentar sus ingresos si tienen mayor experiencia laboral.

Posteriormente, a partir del aparecimiento y la consolidación de la educación en la teoría del capital humano, se tomó a la educación como un factor fundamental para el crecimiento y el bienestar de los países, y la reducción de las desigualdades, ya que esta aporta a la solución de problemas sociales y económicos como la pobreza y el desempleo (Acevedo, Montes, Vásquez, Villegas \& Brito, 2007). Schultz (1961) fue quien estableció por primera vez el término capital humano como sinónimo de educación y formación. Sin embargo, se reconoce que el concepto de capital humano tiene sus orígenes en los trabajos de Becker (1964), en los cuales se refiere al conocimiento, la información, las ideas, las habilidades y la salud de los individuos (Becker, 2002). A pesar de que la educación es considerada el principal productor de capital humano, Becker (1962) advirtió que la inversión en este no solo se 
basa en la educación sino también en otros factores como el entrenamiento laboral, el consumo de vitaminas, en la atención médica e inclusive en la información a la que los individuos tienen acceso.

En este sentido, los jóvenes pertenecientes al grupo nini, al estar fuera del sistema educativo, estarían en desventaja en la adquisición de capital humano con respecto a los jóvenes que sí se encuentran estudiando. Además, representarían un sector que, lejos de aportar al crecimiento económico de un país, comprometería este ámbito debido a que su poca cualificación no les permitiría desempeñar actividades que promuevan una producción de bienes y servicios eficiente.

Una vez establecida la importancia de la educación de los jóvenes, en la siguiente sección se presentan aspectos relacionados con los jóvenes que no estudian ni trabajan.

\section{Los jóvenes ninis}

\subsection{El término nini}

Bell y Thurlby (2017) manifiestan que el término nini es una "caja negra". La complejidad de definir a los ninis y establecer límites entre quiénes son y quiénes no lo son justifica plenamente la analogía de los autores. El término nini ${ }^{4}$ corresponde al equivalente "NEET", acrónimo en inglés de la expresión "not in employment, education or training". Inicialmente, Istance, Rees y Williamson (1994), al realizar un estudio de jóvenes en la ciudad de Glamorgan del Reino Unido, encontraron casos de jóvenes que no buscaban empleo, no estaban educándose o no recibían algún tipo de formación, y denominaron a esta población como "status zero". Posteriormente, el término "NEET" se introdujo formalmente en 1999 en un estudio que tenía como objetivo averiguar las casusas por las que los jóvenes no estudiaban, ni trabajaban, el cual fue elaborado para el Gobierno de ese país. El término NEET surgió con el interés de aclarar el concepto y evitar así connotaciones negativas (Bolaños \& Rivera, 2016).

Este término ha tenido varias críticas, ya que en algunos casos es utilizado para referirse únicamente a los jóvenes rechazados o desertores del sistema

4 La Fundación del Español Urgente (Fundéu) menciona que nini es un neologismo procedente de la expresión "ni estudia ni trabaja", se escribe en una sola palabra, sin espacio ni guion, y no es necesario resaltarlo con comillas ni cursiva. Puede usarse como sustantivo y como adjetivo; además, su plural es ninis, no ni-nis o (los) ni-ni. 
escolar o, en otros casos, se pone más énfasis en la parte laboral, es decir para referirse al desempleo juvenil (Negrete \& Leyva, 2013). Negrete y Leyva (2013) señalan además que, aunque esta palabra no se encuentra expresada de manera oficial como un término estadístico ni se la ha tratado de manera multilateral, se ha vuelto una estadística no oficial, que circula como si lo fuera $\mathrm{y}$, probablemente, ese mismo hecho hace que se refuerce su atractivo.

\section{2. ¿Quiénes forman parte del grupo nini?}

A diferencia del desempleo o el empleo, no existe una norma internacional para definir de forma legítima la tasa de ninis (Elder, 2015). No obstante, la Organización Internacional del Trabajo (OIT, 2012) definió a la tasa de ninis como el porcentaje de jóvenes entre 15 y 24 años que no están en el empleo y que no reciben educación o formación. La misma organización indica que los ninis son una mejor medida del universo actual de jóvenes participantes en el mercado laboral en comparación con la tasa de inactividad de ellos, ya que esta última incluye a aquellos jóvenes que no están en la fuerza laboral y se encuentran estudiando, y que, por tanto, no pueden ser considerados disponibles para el trabajo.

Adicionalmente, definir un concepto internacional para los ninis es complejo puesto que las características de estos jóvenes difieren considerablemente de un país a otro. Por ejemplo, el rango de edad de análisis en países asiáticos, como Japón, va desde los 15 a los 34 años (Kosugi, 2006). Para países europeos, el intervalo varía desde los 15 hasta los 24 o 29 años (ILO, 2017). En Australia, Craig Powell y Brown (2015) expresan que el rango de estudio de los ninis en ese país va desde los 15 hasta los 35 años. Para el caso de América Latina, organismos como la Cepal (2014) y el Banco Mundial (De Hoyos et al., 2016) toman el rango de edad de 15 a 29 años y de 15 a 24 años, respectivamente. Este último rango de edad coincide con la definición de juventud de la Organización de las Naciones Unidas - ONU-.

La oIt (2012) señala que, en algunos países, el grupo de edad de los ninis es seleccionado según el fin de la política a ser aplicada. Por ejemplo, para adolescentes en edad de trabajar de 15 a 17 años y adultos jóvenes entre 18 y 24 años. Con esto, se evidencia tanto la problemática que se genera en cuanto a las comparaciones de porcentajes de jóvenes ninis a nivel de regiones, como también la dificultad para establecer una política única para contrarrestar esta incidencia, pues no está orientada hacia un grupo de personas de la misma franja etaria.

Además de la divergencia relacionada con la edad de los ninis, está la heterogeneidad del grupo. En un informe publicado en el 2012 por la 
Fundación Europea para la Mejora de las Condiciones de Vida y de Trabajo -Eurofound- se diferencian los cinco subgrupos, que se presentan en la tabla 1, como parte de la población nini.

Tabla 1. Subgrupos que conforman la población nini

\begin{tabular}{|c|c|}
\hline Concepto & Descripción \\
\hline Desempleados convencionales & Incluye a los jóvenes desempleados de corto y largo plazo \\
\hline No disponibles & $\begin{array}{l}\text { Incluye a los jóvenes que están implicados en cuidados en el } \\
\text { hogar, tienen responsabilidades familiares, están enfermos o } \\
\text { discapacitados }\end{array}$ \\
\hline Desenganchados & Son los jóvenes que no buscan educarse ni trabajar \\
\hline Buscadores de oportunidades & Jóvenes que buscan trabajo y capacitación \\
\hline Ninis voluntarios & $\begin{array}{l}\text { Jóvenes que realizan otras actividades como viajar o se dedi- } \\
\text { can al arte, la música y el autoaprendizaje }\end{array}$ \\
\hline
\end{tabular}

Fuente: Eurofound (2012)

A partir de la tabla 1 se pueden inferir algunos aspectos. Primero, el grupo nini es bastante heterogéneo y los motivos para conformarlo pueden ir más allá de la voluntad propia, es decir, ser nini no puede ser asociado directamente como el resultado de la falta de empeño o, como manifiestan Málaga et al. (2014), no es sinónimo de "ociosidad". Después, se debe analizar con atención a los jóvenes pertenecientes al grupo de los desenganchados, identificados como pertenecientes al hard core, puesto que además de no hacer nada el deseo de no hacerlo puede acarrear un mayor nivel de vulnerabilidad (Málaga et al., 2014). Finalmente, esta información revela que el diseño de políticas públicas para atender a los jóvenes ninis no puede responder a una receta única. Es necesario indagar en la composición de los subgrupos nini de cada país y entender las causas que motivan su existencia.

\subsection{Factores que inciden en la formación de los ninis}

Para los países europeos existe una caracterización clara de los jóvenes ninis: casi todos son mujeres, pertenecen a familias de migrantes, tienen un bajo o medio nivel de instrucción de educación formal, sus padres tienen bajo nivel de educación, sus familias pertenecen a la clase pobre o rica, y sus padres pueden estar divorciados (Mascherini, 2017). A pesar de que en América Latina la evidencia empírica orientada a la determinación de los factores que podrían influir para que los jóvenes se conviertan en ninis es limitada, esta sección se concentrará en los estudios de la región con el fin de que la 
interpretación de los resultados de las estimaciones pueda ser realizada en un contexto relativamente cercano al del país de análisis, Ecuador.

Entre la evidencia empírica para Latinoamérica, se puede mencionar a Aguayo, Mancha y Rangel (2013) (México), Monteiro (2013) (Brasil) y Málaga et al. (2014) (Perú). Además, existen algunos estudios descriptivos que permiten tener una idea del comportamiento de los jóvenes ninis, los cuales llegan a conclusiones similares. En resumen, factores agregados, como la pobreza, la desigualdad, el desempleo, fallos en el sistema de educación, e individuales, como la edad, la etnia, el género y la renta familiar, serían los determinantes de este fenómeno. Así, se puede concluir que el ser un nini está ligado a la estructura de las desigualdades sociales y constituye un riesgo de exclusión social (Serracant, 2014).

Analizando los factores individuales, O'Dea et al. (2014) indican que, además de que la tasa de ninis aumenta con la edad, son factores demográficos y socioeconómicos los que también influyen. Por ejemplo, las personas del sexo femenino tendrían una mayor probabilidad de ser nini. Ospina, Catagena, García-Suaza, Guataquí y Jaramillo (2017) confirman esa tendencia para el caso colombiano. Sin embargo, este hallazgo contradice los resultados obtenidos para el caso mexicano por Aguayo et al. (2013), autores que sugieren que son los hombres los más propensos a formar parte de los jóvenes ninis.

En cuanto a los factores relacionados con el ambiente familiar, se evidencia que los jóvenes son menos propensos a ser ninis si viven con sus dos padres (Aguayo et al., 2013). Por otro lado, si los padres tienen un alto nivel de instrucción, sus hijos tendrían una probabilidad menor de convertirse en ninis (O’Dea et al., 2014). El nivel de educación de los padres, especialmente el de la madre, podría afectar de manera positiva el rendimiento de los hijos (Guryan, Hurst \& Kearney, 2008). Otro de los factores que incidirían en la formación de los ninis se refiere al nivel de escolaridad del joven, ya que la probabilidad de ser nini disminuye por cada año de educación formal aprobado (Málaga et al., 2014; Aguayo et al., 2013). Esta información se complementa con el informe del Banco Mundial (De Hoyos et al., 2016), en el cual se señala que el nivel de escolaridad de los ninis es bajo. En el 2010, más del $25 \%$ del grupo nini no había terminado la escuela primaria, mientras que otro $43 \%$ había terminado la primaria pero no la secundaria.

En cuanto al lugar donde se ubican los ninis, se reporta que un gran porcentaje se localizan en las áreas urbanas (Bolaños y Rivera, 2016; Cepal, 2014). Adicionalmente, la posibilidad de convertirse en nini se reduciría cuando los ingresos del hogar aumenten, es decir, cuando el entorno socioeconómico de las familias es bueno (Aguayo et al., 2013). 


\subsection{Cómo hacer frente a los jóvenes ninis}

Las consecuencias de la inactividad de los jóvenes ninis, además de estar relacionadas con pobreza y criminalidad, incluyen el costo económico en el que incurren los países. Por ejemplo, según Mascherini (2017), en el 2008 las pérdidas relacionadas con la inactividad laboral de los ninis en la Unión Europea fueron de 147 billones de dólares, valor que representa el 1\% del PIB de la región. En el 2011 este valor ascendió a 187 billones de dólares, representando el 1,2\% del PIB. Estas cifras y las consecuencias sociales de la condición nini fueron el gatillo que impulsó a los países miembros de la Unión Europea a incluir el tema en la agenda política de la región y en el 2014 la Unión Europea implementó el denominado Youth Guarantee, plan que tiene por objetivo garantizar que todos los jóvenes menores de 25 años tengan acceso a una oferta de calidad en: empleo, educación continua, aprendizaje y entrenamiento, en los cuatro meses posteriores de estar desempleado o de haber abandonado la educación formal (Eurofound, 2012). Según el mismo organismo, las medidas implementadas por el Youth Guarantee comprenden la actuación de los estados miembros en seis ejes generales: 1) información, guía y asesoría; 2) programas de difusión; 3) asistencia a los jóvenes en el proceso de transición de la escuela al trabajo; 4) entrenamiento; 5) pasantías; y 6) emprendimiento juvenil. ${ }^{5}$

A pesar de que la iniciativa Youth Guarantee obedece a un acuerdo a nivel de la Unión Europea, los programas puntuales no obedecen a un modelo patrón, debido a la diversidad de los ninis, hecho que no solo dificulta la identificación de los miembros del grupo, sino que también afecta a la hora de pensar en políticas públicas para combatir el problema. En el caso europeo, la Eurofound (2012) reporta cuatro clústeres para caracterizar a los jóvenes ninis de los países europeos, los cuales son mencionados en la tabla 2.

La información de la tabla 2 sugiere un aspecto adicional: el estado de bienestar de cada país también podría influir en el alcance de las medidas para atender a los jóvenes ninis y, por tanto, en las características de los ninis

5 Una de las iniciativas más recientes y que ha llamado la atención es la propuesta por Portugal, país que tiene cerca del $20 \%$ de ninis, y que en el 2017 creó el programa “Empreende Já" (Emprende ya), cuyo objetivo es fomentar el emprendimiento de los jóvenes ninis. Específicamente, se otorgan 700 euros por mes a los jóvenes ninis y este valor debe ser utilizado en el desarrollo de un proyecto que después de seis meses puede ser financiado con fondos no reembolsables. Este proceso cuenta con la orientación y tutoría de especialistas en emprendimiento y proyectos (Emprende Já, 2017). 
Tabla 2. Subgrupos que conforman la población nini

\begin{tabular}{cl}
\hline Clúster & \multicolumn{1}{c}{ Características } \\
\hline 1 & $\begin{array}{l}\text { Conformado por Austria, Dinamarca, Finlandia, Alemania, Luxemburgo, los Países } \\
\text { Bajos, Suecia y el Reino Unido. Muestra tasas bajas de ninis y altas tasas de trabajado- } \\
\text { res inactivos, quienes están poco calificados. }\end{array}$ \\
$2 \quad \begin{array}{l}\text { Formado por Bulgaria, Grecia, Hungría, Italia, Rumania, Polonia y Eslovaquia, con } \\
\text { altas tasas de ninis, especialmente en el grupo de las mujeres, quienes tienen el estatus } \\
\text { de inactivos y sin experiencia laboral. }\end{array}$ \\
$\quad \begin{array}{l}\text { Lo conforman Estonia, Irlanda, Letonia, Lituania, Portugal y España, países que com- } \\
\text { parten afectaciones económicas asociadas con crisis financieras. Sus tasas de ninis son } \\
\text { altas y la mayoría de ninis son hombres que, a pesar de estar desempleados, tienen } \\
\text { experiencia laboral y están altamente cualificados. }\end{array}$ \\
$\begin{array}{l}\text { Integrado por Bélgica, Chipre, la República Checa, Francia y Eslovenia, con tasas por } \\
\text { debajo de la media europea de ninis. Los ninis de estos países están registrados como } \\
\text { desempleados y tienen experiencia laboral y un nivel de instrucción medio. }\end{array}$ \\
\hline
\end{tabular}

Fuente: Eurofound, 2012

de los diferentes países. Mascherini (2017), autor que analizó las características de los ninis considerando el modelo de estado de bienestar ${ }^{6}$ de cada país, confirma la existencia de grupos ninis heterogéneos en los diferentes clústeres de estado de bienestar. Entre los resultados del autor, que permiten establecer estas diferencias, se puede mencionar que no existe distinción entre los jóvenes ninis y no ninis del clúster escandinavo. Según el mismo autor, esto estaría asociado con el hecho de que un estado de bienestar maduro e inclusivo ofrece mayores posibilidades de atender a los jóvenes vulnerables y, sobre todo, de no marginalizarlos. Adicionalmente, la educación sería el principal predictor de la probabilidad de ser nini, siendo los jóvenes de baja y alta renta más propensos a serlo con respecto a los jóvenes con renta media. El efecto del ingreso sería mayor en los países liberales mientras que en los clústeres mediterráneo y universal el efecto sería limitado. Finalmente, en el caso de la educación, reportado como el factor que más explica la probabilidad de ser nini, los jóvenes con bajo nivel de escolaridad tendrían más probabilidad de convertirse en ninis si son comparados con los jóvenes que alcanzaron la educación secundaria o superior. No obstante, este efecto

6 El análisis fue realizado según la clasificación hecha por Marshall (1950), quien propone cinco clústeres: 1) universal: formado por Dinamarca, Finlandia y Suecia; 2) liberal: Irlanda y Reino Unido; 3) mediterráneo: Chipre, España, Grecia, Italia, Malta y Portugal; 4) centrados en el empleo: Austria, Bélgica, Alemania, Francia, Luxemburgo y Países Bajos; 5) postsocialistas: Bulgaria, República Checa, Estonia, Hungría, Lituania, Letonia, Polonia, Rumanía, Eslovenia y Eslovaquia. 
también sería más fuerte en los países liberales, mientras que en los países mediterráneos sería poco relevante. Así, se puede concluir que las políticas para hacer frente a la problemática nini necesariamente deben ser variadas, y su diseño y efectividad puede diferir según el nivel de madurez del estado de bienestar de cada país.

En este contexto, cabe un interrogante relacionado con los jóvenes ninis de la región latinoamericana: ¿pueden los regímenes de bienestar de la región hacer frente a la problemática nini? Primero, nótese que en este caso se sustituyó la palabra estado por régimen. Esta sutileza en la terminología, sugerida por Martínez (2007), es para llamar la atención en el sentido de que los sistemas de bienestar latinoamericanos presentan un alto grado de informalidad e intervenciones públicas no robustas, y dependen en gran medida del ámbito doméstico; es decir no se podría invocar a un estado de bienestar en el estricto sentido de lo que esto significa. Estas características generales de los regímenes latinoamericanos que, según reporta la autora, están cambiando como respuesta a las exigencias sociales de la época que de alguna $u$ otra forma intentan reivindicar una mayor respuesta estatal para hacer frente a los riesgos en cualquier etapa de la vida, niñez, adolescencia, adultez o tercera edad, también sugieren una respuesta para la pregunta planteada que dista de ser optimista, y revelan que el impacto de los perjuicios de corto y largo plazo asociados con la inactividad de los jóvenes ninis pueden ser más fuertes en América Latina.

\subsection{Politicas para reducir el flujo de nuevos ninis}

A pesar de la complejidad de uniformizar las medidas para hacer frente a los jóvenes ninis, esta sección tiene como objetivo realizar una descripción general de las políticas implementadas, que se pueden dividir en políticas para evitar el abandono escolar, políticas para la inserción laboral y programas. Cada uno de estos aspectos es descrito a continuación.

Entre las políticas para evitar el abandono escolar de los jóvenes, están las intervenciones por el lado de la demanda, que abarcan las transferencias monetarias condicionadas, los programas de becas y las intervenciones para informar a los estudiantes sobre los beneficios de la educación; mientras, las intervenciones, por el lado de la oferta, abarcan las maneras de mejorar la calidad de la educación, en particular para los jóvenes en riesgo (Almeida, Fitzsimons \& Rogers, 2015).

El objetivo de las transferencias de renta es reducir la pobreza y aumentar el capital humano de los infantes. Para alcanzar estos objetivos, las transferencias se dan a los hogares pobres, y son condicionadas a la inversión en 
educación, salud y nutrición de sus hijos. Por ejemplo, México cuenta con el programa de transferencias condicionadas contra la pobreza llamado Oportunidades, el cual otorga una transferencia a los padres de áreas rurales para enviar a sus hijos a la escuela (Chiapa \& Juárez, 2012). Este programa ha tenido buenos resultados debido a que las transferencias monetarias están condicionadas a una serie de comportamientos destinados a proteger y desarrollar el capital humano de las familias pobres. Otro tipo de intervención se da a través de becas al mérito, que son otorgadas con el objeto de disminuir la tasa de deserción en el bachillerato. Por ejemplo, en Israel un programa de desarrollo que otorgaba incentivos a los alumnos por aprobar un curso y por finalizar la secundaria hizo que aumentara significativamente la tasa de graduación de las mujeres en un 10\% (Angrist \& Lavy, 2009).

En lo que respecta a las intervenciones de información, estas hacen referencia a la información que se proporciona a los jóvenes sobre los retornos de la educación para que, de esta manera, se sientan motivados a continuar con sus estudios y no abandonen el sistema escolar. En cuanto a este tipo de intervención, se puede reportar los resultados que se obtuvieron en República Dominicana, en donde entre el $80 \%$ y el $90 \%$ de los jóvenes completaba la escuela primaria, y de ellos solo entre el $25 \%$ y $30 \%$ terminaba la escuela secundaria. Jensen (2010) explica que, al informar a los jóvenes sobre cuáles podrían ser los ingresos a futuro, estos podrían aumentar sus años de educación formal, entre 0,20 y 0,35 años más. Sin embargo, hay que tener en cuenta que se podría combinar las intervenciones de información con las transferencias monetarias, ya que precisamente son los hogares más pobres los que tienen menor información acerca de cuáles son los retornos de la educación (Barr, 2012).

Por el lado de la oferta, las intervenciones socioemocionales tienen como objetivo captar las habilidades no cognitivas, como rasgos de la personalidad, metas, el carácter y las motivaciones, que son valoradas en el mercado de trabajo y en la escuela (Kautz, Heckman, Diris, ter Weel \& Borghans, 2014). De Hoyos et al. (2016) explican la importancia de las intervenciones socioemocionales para fortalecer las aptitudes de este tipo, y así reducir el abandono escolar y otras conductas de riesgo entre los jóvenes durante el bachillerato. Un ejemplo es la evaluación de programas de apoyo a los jóvenes en riesgo en Chicago, la cual muestra que, según Heller, Pollack, Ander y Ludwig (2013), durante el primer año de implementación del programa disminuyeron los arrestos por violencia en un 44 \% y mejoró, de forma sustancial, la asistencia a clases, el promedio general de las calificaciones y la permanencia de los jóvenes en la escuela. 
Otra propuesta son los sistemas de detección temprana. Estas intervenciones son eficientes si son correctamente focalizadas y se logran efectuar a una edad temprana, ya que de esta manera se identifican los grupos de riesgo, como los que tienen bajas calificaciones o jóvenes que no asisten a clases, y así se logra prevenir el abandono escolar (Maguire, 2013). Finalmente, se menciona la intervención temprana como mecanismo para promover la asistencia escolar, reducir la delincuencia, fomentar la productividad en el trabajo y reducir el embarazo de adolescentes (Currie y Almond, 2011). El Proyecto Perry en Estados Unidos mostró que la intervención temprana incrementa las probabilidades de graduación del bachillerato en $17 \%$.

Estas no serían las únicas propuestas de intervención para controlar el problema de los jóvenes nini. Maguire (2013) señala como políticas para prevenir el aumento de ninis las siguientes: 1) la introducción de planes de estudios alternativos dentro de la escuela con el objeto de que esta brinde educación más profesional y técnica, y trabajar en asociación con otras organizaciones, como colegios técnicos especializados, para combatir la propensión al abandono prematuro; y 2) el aumento de la edad de participación de los jóvenes en el sistema educativo, es decir aumentar la edad obligatoria de educación. Esta política es promulgada principalmente en el Reino Unido, pese a que corre el riesgo de ser simplemente una manera de "almacenamiento" de jóvenes, si no va acompañada con el aprendizaje significativo que esté acorde con el mercado laboral.

En lo que se refiere a las políticas para favorecer la inserción de los jóvenes en el mercado laboral, se deben abordar los programas de capacitación enfocados en habilidades académicas generales, habilidades técnicas específicas o aptitudes socioemocionales, ya sea en centros educativos o en el trabajo, para forjar el capital humano de los ninis (De Hoyos et al., 2016, p. 45): “La hipótesis en la que se basan estos programas es que para que los empleadores estén dispuestos a contratar ninis, los jóvenes necesitan ciertas habilidades para alcanzar mayores niveles de productividad". No obstante, los gobiernos también pueden ayudar a los jóvenes en su inserción en el mercado formal de trabajo, con el otorgamiento de subsidios salariales o creando programas de emprendimiento para que logren emplearse por cuenta propia. Otra de las opciones se refiere a la aplicación de programas de empleo público que permitan facilitar el ingreso de los jóvenes al mercado laboral, y otorgar servicios públicos de empleo que permitan dar a conocer sobre vacantes basándose en la ubicación, habilidades y preferencias de los jóvenes.

Finalmente, con respecto a los programas, con el fin de capacitar a los jóvenes en las distintas áreas de trabajo, ciertos países ofrecen programas de 
capacitación para los jóvenes ninis. Por ejemplo, diversos países latinoamericanos, con el apoyo de organismos internacionales, han creado sus propios programas, que consisten en la aplicación de cursos que permitan capacitar a la mano de obra juvenil para que logren insertarse en el mercado. Específicamente, en el 2012, el Banco Interamericano de Desarrollo — BID- creó en Cartagena (Colombia) la alianza Nuevas Oportunidades de Empleo - NEOcon el objetivo de formar a los jóvenes para el trabajo. En este programa los jóvenes cuentan con aprendizaje en línea, fortalecimiento técnico de la educación secundaria y mejoras en la orientación vocacional e inserción laboral. La iniciativa se aplica en Argentina, Brasil, Colombia, República Dominicana, El Salvador, México, Panamá, Paraguay, Perú y Uruguay.

\section{Datos y metodología}

\subsection{Datos}

En este estudio, que tiene como objetivo determinar los factores que afectan la formación de los jóvenes ninis en el Ecuador, se utiliza la Encuesta de Condiciones de Vida — ECV — realizada por el Instituto Nacional de Estadísticas y Censos - INEC - Esta permite obtener un panorama sobre el nivel de vida y el bienestar de la población ecuatoriana. El universo de la encuesta constituyen todos los hogares de las áreas urbanas y rurales del país. Los datos analizados en el presente artículo corresponden a la sexta ronda de la ECV, 2014 , e incluye a 28.970 hogares, con un total de 109.694 individuos. Para este estudio, únicamente se considerará al grupo de jóvenes de 15 a 24 años de edad, con un total de 18.992 observaciones.

Para los propósitos de este artículo, el grupo nini está formado por los jóvenes que no están trabajando (desempleados e inactivos), ni estudiando. Sin embargo, a efectos de extraer información más detallada, también se realizará un análisis distinguiendo tres casos: 1) desempleados convencionales (conforman el $1 \%$ de la muestra); 2) no estudian ni trabajan pero desean trabajar (15\% de la muestra); y 3) no estudian ni trabajan ni desean hacerlo (84\%). La elección de estos grupos de análisis fue realizada considerando la información de la tabla 1, que sugiere que el análisis del grupo nini debe ser separado considerando el nivel de la "gravedad" de la inactividad.

Según la información proporcionada por la ECV, en el Ecuador la población de jóvenes de 15 a 24 años ha ido aumentando año a año, como se ve en la tabla 3 . 
Tabla 3. Número de jóvenes por grupos de edad (1999-2014)

\begin{tabular}{lccc}
\hline \multicolumn{1}{c}{ Año } & \multicolumn{1}{c}{1999} & 2006 & \multicolumn{1}{c}{2014} \\
\hline Total población & 10.654 .175 & 13.278 .797 & 15.957 .336 \\
\hline $15-24$ años & 2.092 .255 & 2.423 .886 & 2.758 .102 \\
\hline $15-19$ años & 1.128 .415 & 1.304 .979 & 1.509 .812 \\
\hline 20-24 años & 963.840 & 1.118 .907 & 1.248 .290 \\
\hline
\end{tabular}

Fuente: elaboración propia con base en INEC, 1999, 2006, 2014

Siendo el grupo de interés de este artículo los jóvenes que ni estudian ni trabajan y están entre los 15 y los 24 años de edad, se pudo determinar que para 1999 existían 410.108 ninis, es decir $20 \%$ de los jóvenes pertenecían a dicha población. En el 2006, esta cifra aumentó a 436.598 jóvenes, que representan el 18,1\%, y, para el 2014, existían alrededor de 510.896 jóvenes, que representan el $18,5 \%$.

$\mathrm{Al}$ analizar la distribución de los ninis por rangos de edad, en la figura 1 se observa que la mayor concentración de ninis se da entre los 20 y los 24 años, lo cual probablemente quiere decir que los jóvenes en este rango de edad no han ingresado o abandonaron los estudios universitarios y no cuentan con un empleo.

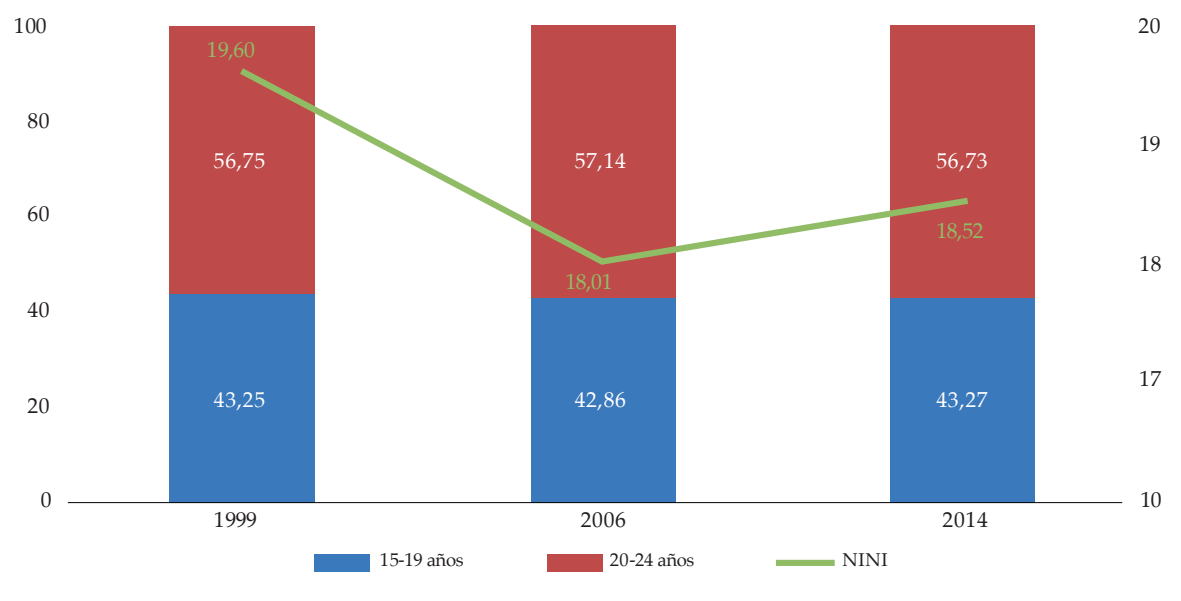

Figura 1. Tasa de jóvenes ninis por grupos de edad (1999-2014)

Fuente: elaboración propia con base en INEC, 1999, 2006, 2014

Lo anterior puede estar relacionado, principalmente, con el hecho de que en los años universitarios hay mayor deserción escolar, especialmente en los primeros semestres de la carrera universitaria (Ramírez \& Corvo, 2007). Según 
Rodríguez y Vindas (2011), esto se debe sobre todo a factores económicos, falta de disciplina y a la carga académica. En la parte laboral, en esta edad los jóvenes no cuentan con las suficientes habilidades y difícilmente podrán encontrar un empleo (Brada, Marelli \& Signorelli, 2014).

Los resultados por sexo aparecen en la figura 2. Para el 2014, el 28,3\% de los jóvenes ninis son hombres y el 71,7\% son mujeres, resultados que siguen la tendencia presentada por el Banco Mundial (De Hoyos et al., 2016), en donde se menciona que la proporción de ninis mujeres corresponde a los dos tercios de jóvenes que conforman dicho grupo.

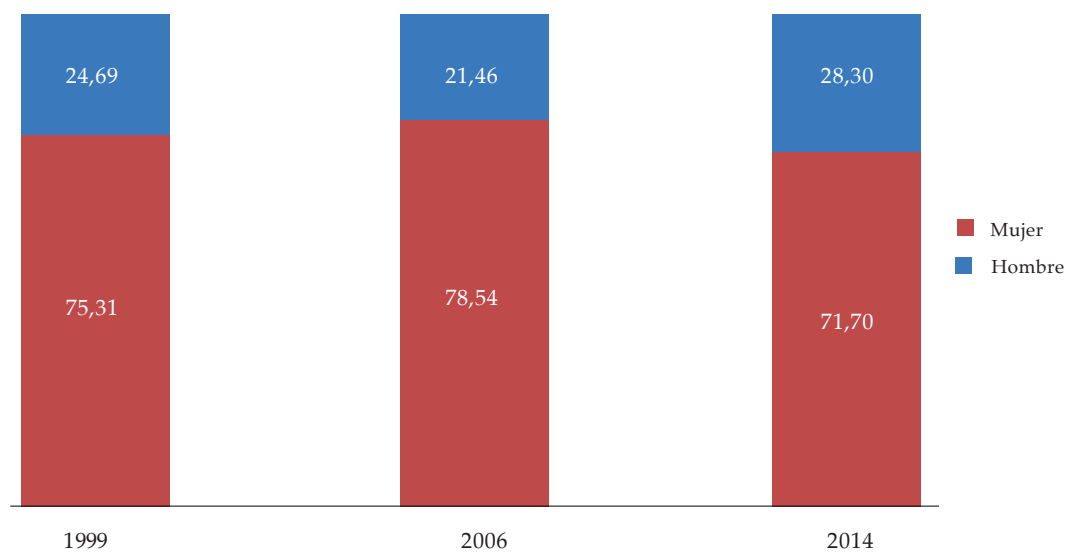

Figura 2. Tasa de jóvenes ninis por sexo (1999-2014)

Fuente: elaboración propia con base en INEC, 1999, 2006, 2014

Para el análisis del estado civil, se construyeron las siguientes categorías: solteros, si tiene algún tipo de compromiso (casados y unión libre) y si han tenido un compromiso (separado, divorciado y viudo). En la figura 3 se puede observar que, en el 2014, el 53,03\% de los ninis se encontraba soltero, el $41,72 \%$ casado y el 5,25\% separado; es decir, la mayor proporción de jóvenes ninis se evidencia en los solteros. Sin embargo, a través de los años se puede notar que los ninis separados han ido en aumento.

En cuanto a la etnia, se puede observar en la figura 4 que la mayor concentración de ninis se encuentra en los mestizos con el 77,42\%, obviamente por ser la mayoría a nivel poblacional, seguido por los jóvenes afrodescendientes y montuvios ${ }^{7}$ con el 7,60 \% y el 6,55\%, respectivamente. Además, se evidencia

7 El término montuvio, reconocido por la Real Academia Española, se utiliza en Ecuador para referirse a los campesinos que habitan en la Costa ecuatoriana. 
el 4,92\% para los indígenas y un reducido porcentaje del 3,52\% para el caso de los blancos.

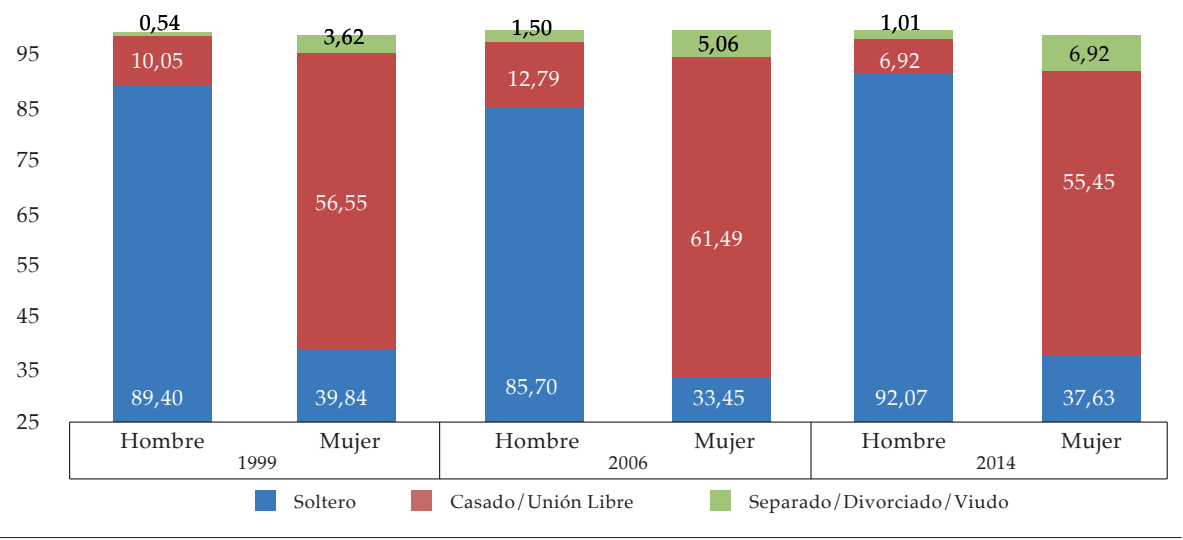

Figura 3. Jóvenes ninis por estado civil, 1999-2014

Fuente: elaboración propia con base en INEC, 1999, 2006, 2014

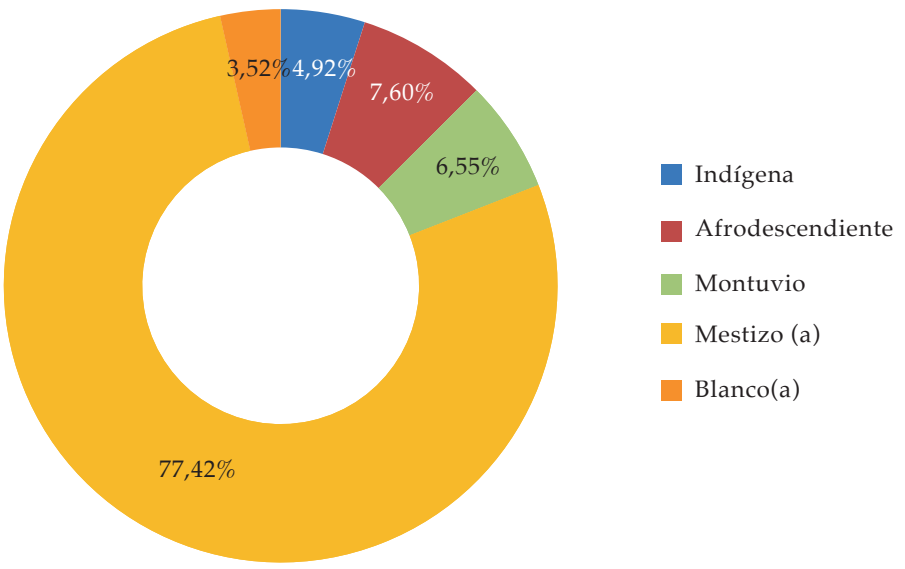

Figura 4. Jóvenes ninis por etnia, 1999-2014

Fuente: elaboración propia con base en INEC, 2014

En cuanto a la localización geográfica, para el 2014, de los 510.896 jóvenes ninis, el 71,04 \% se encontraba situado en el área urbana y el 28,96\% en el área rural. Además, en la figura 5 se puede evidenciar el constante crecimiento que ha tenido la concentración de jóvenes ninis en el área urbana desde 1999. Estos resultados siguen el mismo patrón mostrado para toda Latinoamérica (De Hoyos et al., 2016). 


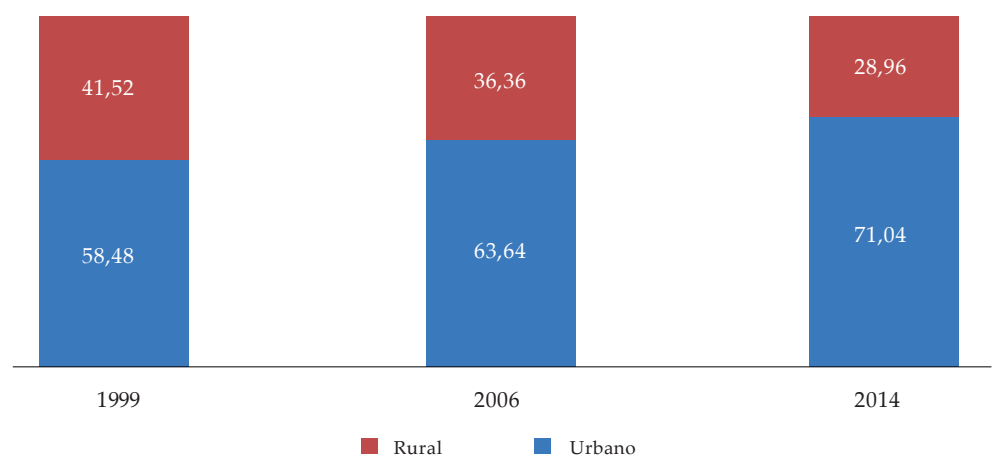

Figura 5. Jóvenes ninis por área, 1999-2014

Fuente: elaboración propia con base en INEC, 1999, 2006, 2014

Analizando la distribución de ninis por sexo dentro de las respectivas áreas geográficas, $80,72 \%$ de los ninis hombres se ubica en la zona urbana y el restante $19,28 \%$ en la zona rural; por su parte, para las jóvenes mujeres ninis el 67,23\% está en el área urbana y en el área rural el 32,77\%. En la figura 6, se puede ver la distribución de los jóvenes ninis a nivel de regiones, ${ }^{8}$ observándose que una mayor concentración de este grupo se encuentra en la Costa con un 60,31\%, seguido de la Sierra con un $34,5 \%$.

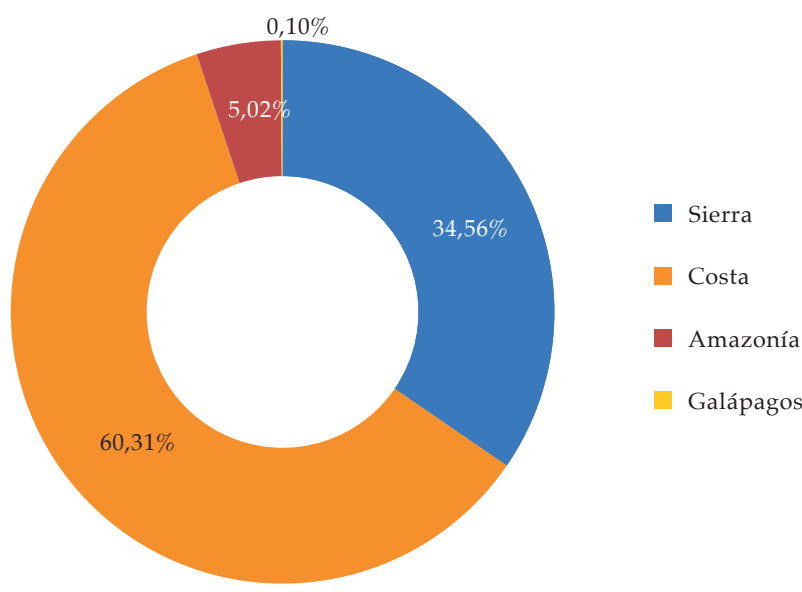

Figura 6. Jóvenes ninis por región, 2014

Fuente: elaboración propia con base en INEC, 2014

8 El Ecuador está dividido en cuatro regionales naturales: Amazonía, Costa, Sierra y Galápagos. 
En la figura 7, se puede observar que los resultados anteriores se confirman al realizar un análisis por provincias ${ }^{9}$ del Ecuador. Así, se puede ver que las provincias que cuentan con la mayor proporción de jóvenes ninis son: Santa Elena (26,34\%), Guayas (23,41\%), Los Ríos (23,33\%), Esmeraldas $(22,81 \%)$ y Manabí (21,55\%). Por su parte, las provincias que cuentan con la menor proporción son: Cotopaxi (10,7\%), Tungurahua (7,89\%) y Chimborazo $(5,84 \%)$. Estos porcentajes son calculados con base en el total de la población de jóvenes de entre 15 y 24 años de edad correspondiente a cada provincia.

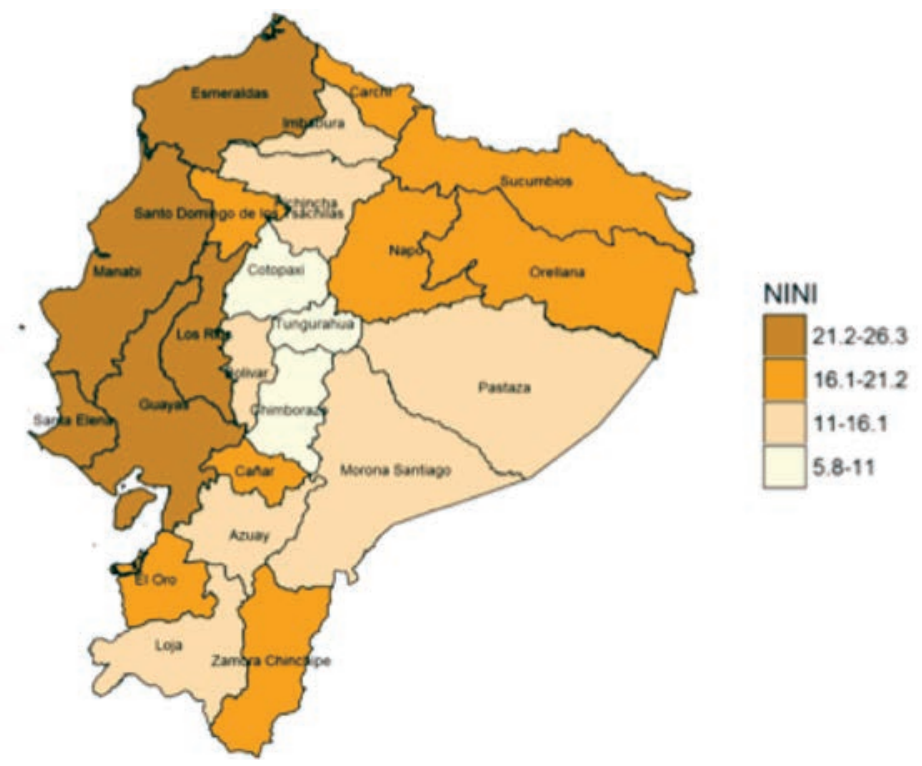

Figura 7. Jóvenes ninis por provincia

Fuente: elaboración propia con base en INEC, 2014

En cuanto a los ingresos, en la figura 8 se puede observar que la mayor concentración de este grupo se encuentra en el quintil 3, que abarca un 23,98\% del total de ninis. Además, se observa en la misma figura que el porcentaje de ninis en los cuatro primeros quintiles gira en torno al $20 \%$, mientras que en el último quintil este porcentaje equivale al $11 \%$. Es decir, la falta de recursos económicos podría incidir en que los jóvenes tengan que abandonar sus estudios con el objeto de encontrar un empleo, pero al no existir oportunidades dentro del mercado laboral, se convierten en ninis.

9 Una provincia es una división político-administrativa conformada por la unión de dos o más ciudades. En la actualidad el Ecuador tiene 24 provincias. 


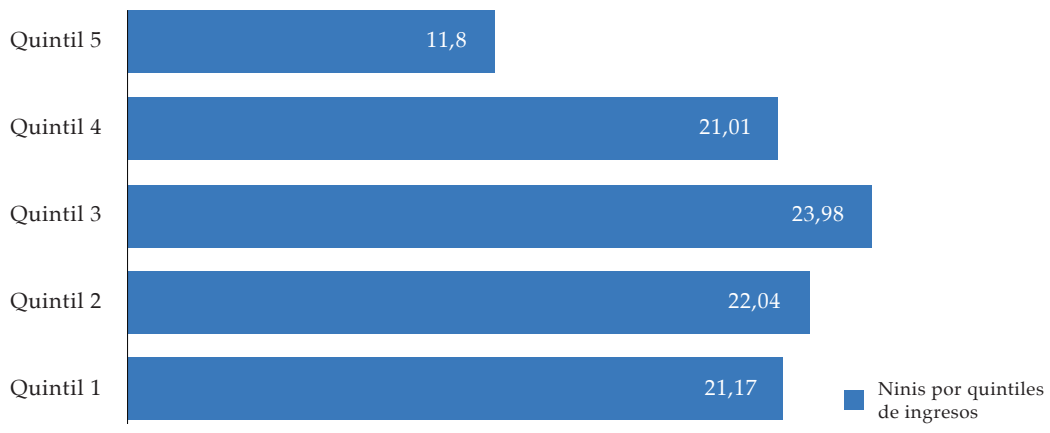

Figura 8. Jóvenes ninis por quintil de ingreso

Fuente: elaboración propia con base en INEC, 2014

Por otro lado, la tasa de discapacidad de los jóvenes ninis es del 5,45\%. Dentro de este subgrupo se encuentran los jóvenes cuya discapacidad es de tipo intelectual (53\%), físico-motora (25\%), visual (5\%), auditiva (8\%) y mental ( $9 \%)$, la cual se refiere a enfermedades psiquiátricas.

Finalmente, al hablar de las características de los padres de los jóvenes ninis, 49,10\% de estos no viven con su madre, mientras que el 64,01\% no viven con su padre. Además, con respecto al nivel de instrucción de los padres, se puede ver en la figura 9 que la mayoría ha cursado únicamente la educación básica, siendo el 59,25\% para el caso de la madre y el 62,57\% para el caso del padre. Le sigue la educación secundaria con el 25,13 \% y 23,50\% para la madre y el padre, respectivamente.

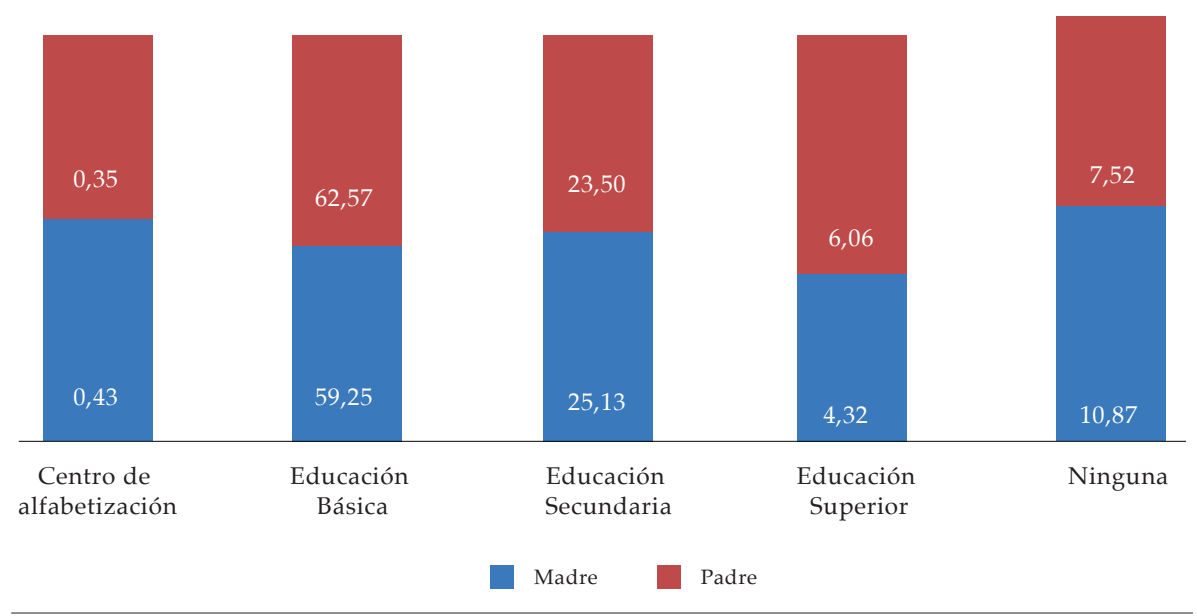

Figura 9. Nivel de instrucción de los padres de los jóvenes ninis

Fuente: elaboración propia con base en INEC, 2014 
Una vez caracterizados los jóvenes ninis del Ecuador, se analizarán las razones por las cuales dejan los estudios. Así, para los jóvenes de 15 a 19 años, las razones principales del abandono escolar son, para el caso de los hombres, los problemas familiares $(19,71 \%)$, las malas calificaciones $(18,28 \%)$ y la falta de dinero (15,65\%). Para el caso de las mujeres las razones de abandono escolar, y en un porcentaje mayor al de los hombres, son principalmente los problemas familiares $(29,48 \%)$, seguido del temor a los maestros $(14,48 \%)$ y los embarazos (14,08\%). Estos resultados se muestran en la tabla 4.

Tabla 4. Razones de abandono del sistema educativo en los jóvenes de 15 a 19 años por sexo

\begin{tabular}{lclc}
\hline \multicolumn{1}{c}{ Hombre } & \multicolumn{2}{c}{ Mujer } \\
\hline Problemas familiares & $19,71 \%$ & Problemas familiares & $29,48 \%$ \\
Malas calificaciones & $18,28 \%$ & Por temor a los maestros & $14,48 \%$ \\
Falta de dinero & $15,65 \%$ & Embarazo & $14,08 \%$ \\
No le interesa & $11,95 \%$ & Trabajo & $10,55 \%$ \\
Enfermedad & $5,21 \%$ & Enfermedad & $7,46 \%$ \\
Por temor a los maestros & $4,84 \%$ & Labores domésticas & $6,96 \%$ \\
Trabajo & $3,46 \%$ & Malas calificaciones & $3,82 \%$ \\
\hline
\end{tabular}

Fuente: elaboración propia con base en INEC, 2014

Por otro lado, para los jóvenes de 20 a 24 años, los motivos de abandono del sistema educativo se mencionan a continuación: para el caso de los hombres son los problemas familiares $(45,63 \%)$, por trabajo $(38,27 \%)$ y debido a las malas calificaciones (11,47\%). En el caso de las mujeres, la principal causa son los embarazos $(68,37 \%)$, seguido por las labores domésticas $(13,90 \%)$ y problemas familiares $(8,86 \%)$.

En general, los jóvenes de 15 a 24 años manifiestan que la primera razón para dejar los estudios, tanto hombres como mujeres, son los problemas familiares, con 25,38\% y 25,66\%, respectivamente; seguido de las malas calificaciones $(16,79 \%)$ y la falta de dinero (12,23\%) en los hombres; y para las mujeres el embarazo $(24,14 \%)$ y el temor a los maestros $(11,80 \%)$. Por último, para ambos sexos la cuarta razón es por motivos de trabajo, con 11,07\% en los hombres y $8,60 \%$ en las mujeres. No obstante, después de abandonar sus estudios, el joven se convierte en nini al no buscar empleo.

Los motivos para esto también varían dependiendo del sexo y del rango de edad del que son parte. Por ejemplo, la tabla 5 señala que las razones por las que los jóvenes ninis de 15 a 19 años no buscan empleo son, para el caso 
de los hombres, porque no tienen necesidad o deseos de trabajar ( $39,68 \%)$, se encuentran enfermos o discapacitados (14,53\%), y en las mujeres su cónyuge o algún familiar no les permiten $(50,74 \%)$, no tienen necesidad o deseos de trabajar $(20,73 \%)$, y como tercera razón, para hombres y mujeres, porque no tienen tiempo, con $14,05 \%$ y $14,13 \%$, respectivamente.

Tabla 5. Razones por las que no buscan empleo los jóvenes ninis de 15 a 19 años por sexo (2014)

\begin{tabular}{lrlc}
\hline \multicolumn{1}{c}{ Hombre } & & \multicolumn{2}{c}{ Mujer } \\
\hline $\begin{array}{l}\text { No tiene necesidad o deseos de } \\
\text { trabajar }\end{array}$ & $39,68 \%$ & $\begin{array}{l}\text { Su cónyuge o su familia no le } \\
\text { permiten }\end{array}$ & $50,74 \%$ \\
Está enfermo/incapacitado & $14,53 \%$ & $\begin{array}{l}\text { No tiene necesidad o deseos de } \\
\text { trabajar }\end{array}$ & $20,73 \%$ \\
No tiene tiempo & $14,05 \%$ & No tiene tiempo & $14,13 \%$ \\
$\begin{array}{l}\text { Su cónyuge o su familia no le } \\
\text { permiten }\end{array}$ & $10,08 \%$ & Está enfermo/incapacitado & $6,19 \%$ \\
No cree poder encontrar & $9,77 \%$ & No cree poder encontrar & $4,12 \%$ \\
No está en edad de trabajar & $4,17 \%$ & No está en edad de trabajar & $1,65 \%$ \\
\hline
\end{tabular}

Fuente: elaboración propia con base en INEC, 2014

Dentro del grupo de ninis de 20 a 24 años, las razones son las mismas que para los de 15 a 19 años, pero con variaciones en los porcentajes. Con respecto a los hombres, el no tener necesidad o deseos de trabajar desciende al 33,23\%, estar enfermo o incapacitado aumenta al 23,85\% y el no tener tiempo pasa al 11,55\%; por su parte, en las mujeres que su cónyuge o familiar no le permitan asciende al 69,02\% y el no tener deseos de trabajar y no tener tiempo descienden al 12,56\% y el 10,82\%, respectivamente.

En general, los jóvenes de 15 a 24 años no buscan empleo, en el caso de los hombres, por no tener necesidad o deseos de trabajar $(37,18 \%)$ y por no tener tiempo (13,08\%). En cambio, para las mujeres, el 61,56\% manifiesta que su cónyuge o familiar no le permite y el 12,17\% se refiere a falta de tiempo.

Finalmente, en la figura 10 se muestra la distribución de la población de jóvenes de 15 a 24 años con las distintas combinaciones entre educación y empleo. Cabe mencionar que el porcentaje de jóvenes que no trabajan pero se encuentran estudiando aumentó entre 1999 y el 2014, lo cual refleja que se está trabajando en el capital humano joven y productivo para las siguientes generaciones. También aparece el porcentaje de jóvenes que sí trabajan y sí estudian, "sisis", con una aparente disminución en el 2014. 


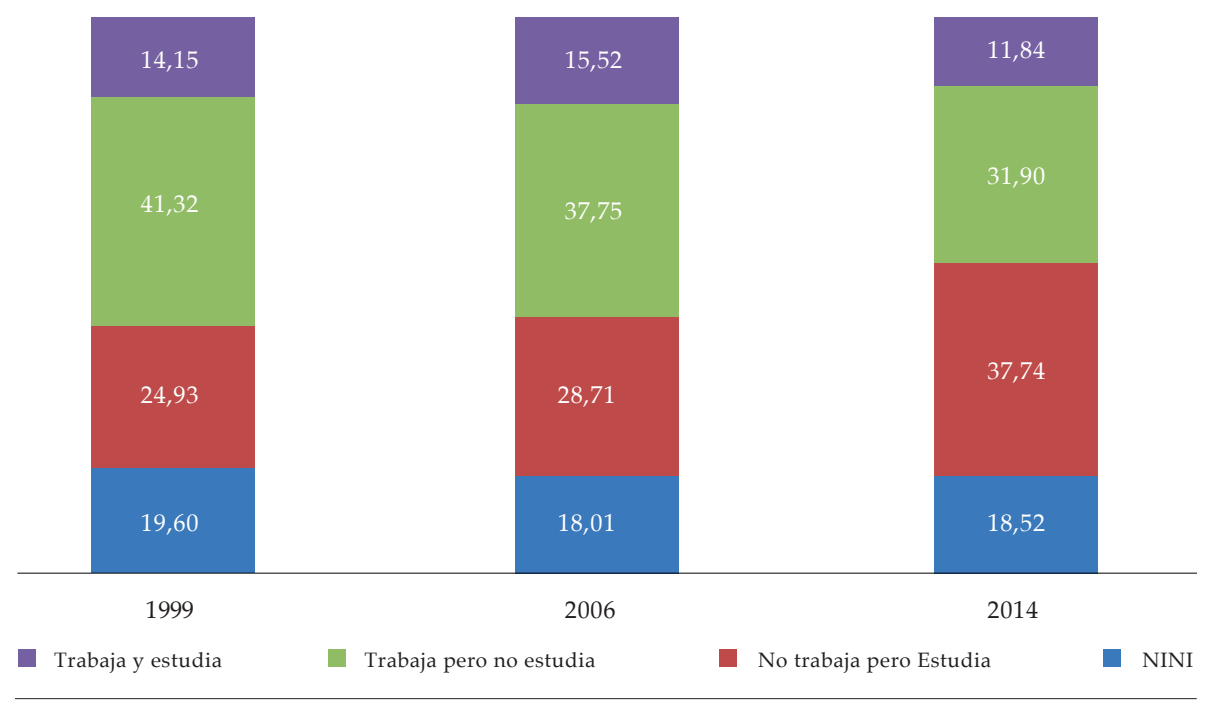

Figura 10. Distribución de la población de jóvenes de 15 a 24 años por empleo y educación, 1999-2014

Fuente: elaboración propia con base en INEC, 2014

\subsection{Metodología}

Con el fin de determinar cuáles son los factores que influyen en la formación de los jóvenes ninis, y siguiendo la literatura relacionada con el tema, como el caso de Aguayo et al. (2013) y Málaga, Oré \& Tavera (2014) que plantearon un modelo para México y Perú, respectivamente, se estimará un modelo logit considerando la siguiente especificación:

nini $_{i}=\beta_{0}+\beta_{1} X_{i 1}+\beta_{2} X_{i 1}^{2}+\beta_{3} X_{i 3}+\beta_{4} X_{i 4}+\beta_{5} X_{i 5}+\beta_{6} X_{i 6}+\beta_{7} X_{i 7}+\beta_{8} X_{i 8}+\beta_{9} X_{i 9}+\varepsilon_{i}$

donde:

$X_{1}$ : edad; $X_{2}$ : área urbana; $X_{3}$ : sexo; $X_{4}$ : estado civil; $X_{5}$ : discapacidad; $X_{6}$ : etnia; $X_{7}$ : presencia de la madre en el hogar; $X_{8}$ : nivel de instrucción; $X_{9}$ : quintil de ingresos; $\varepsilon$ : término de error

A continuación se presenta la descripción de las variables:

\subsubsection{Variable dependiente}

La variable toma el valor 1 si el joven es nini y 0 en caso contrario. 


\subsubsection{Variables independientes}

La tabla 6 muestra las variables tanto socioeconómicas como demográficas que, según la teoría económica y la evidencia empírica revisadas, serán incluidas en el modelo econométrico.

Tabla 6. Variables independientes

\begin{tabular}{|c|c|}
\hline Variable & Descripción \\
\hline $\begin{array}{l}\text { Nini = jóvenes entre } 15 \text { y } 24 \text { años } \\
\text { que no estudian ni trabajan }\end{array}$ & $\begin{array}{l}1=\text { si el joven de } 15 \text { a } 24 \text { años es nini } \\
0=\text { caso contrario }\end{array}$ \\
\hline Edad & Edades de todos los jóvenes entre 15 y 24 años \\
\hline Edad_cuadrado & $\begin{array}{l}\text { Edades de todos los jóvenes entre } 15 \text { y } 24 \text { años elevadas al } \\
\text { cuadrado }\end{array}$ \\
\hline Urbano_5000 & $\begin{array}{l}1=\text { si el joven vive en el área urbana } \\
0=\text { caso contrario }\end{array}$ \\
\hline Hombre & $\begin{array}{l}1=\text { si el joven es hombre } \\
0=\text { caso contrario }\end{array}$ \\
\hline Casado & $\begin{array}{l}1=\text { si el joven es casado } \\
0=\text { otros casos }\end{array}$ \\
\hline Separado & $\begin{array}{l}1=\text { si el joven es separado } \\
0=\text { otros casos }\end{array}$ \\
\hline Discapacidad & $\begin{array}{l}1=\text { si el joven tiene alguna discapacidad } \\
0=\text { caso contrario }\end{array}$ \\
\hline Indígena & $\begin{array}{l}1=\text { si el joven se considera indígena } \\
0=\text { otros casos }\end{array}$ \\
\hline Afrodescendiente & $\begin{array}{l}1=\text { si el joven se considera afrodescendiente } \\
0=\text { otros casos }\end{array}$ \\
\hline Montuvio & $\begin{array}{l}1=\text { si el joven se considera montuvio } \\
0=\text { otros casos }\end{array}$ \\
\hline Blanco & $\begin{array}{l}1=\text { si el joven se considera blanco } \\
0=\text { otros casos }\end{array}$ \\
\hline Madre_hogar & $\begin{array}{l}1=\text { si la madre del joven vive en el hogar } \\
0=\text { caso contrario }\end{array}$ \\
\hline Ninguna & $\begin{array}{l}1=\text { si el joven no tiene algún tipo de educación } \\
0=\text { otros casos }\end{array}$ \\
\hline Alfabetización & $\begin{array}{l}1=\text { si el joven ha cursado algún centro de alfabetización } \\
0=\text { otros casos }\end{array}$ \\
\hline Básica & $\begin{array}{l}1=\text { si el joven tiene educación básica } \\
0=\text { otros casos }\end{array}$ \\
\hline
\end{tabular}




\begin{tabular}{|c|c|}
\hline Variable & Descripción \\
\hline Secundaria & $\begin{array}{l}1=\text { si la madre del joven tiene educación secundaria } \\
0=\text { otros casos }\end{array}$ \\
\hline Superior & $\begin{array}{l}1=\text { si la madre del joven tiene educación superior } \\
0=\text { otros casos }\end{array}$ \\
\hline Quintil_2 & $\begin{array}{l}1=\text { si el joven pertenece al quintil de ingresos } 2 \\
0=\text { caso contrario }\end{array}$ \\
\hline Quintil_3 & $\begin{array}{l}1=\text { si el joven pertenece al quintil de ingresos } 3 \\
0=\text { otros casos }\end{array}$ \\
\hline Quintil_4 & $\begin{array}{l}1=\text { si el joven pertenece al quintil de ingresos } 4 \\
0=\text { caso contrario }\end{array}$ \\
\hline Quintil_5 & $\begin{array}{l}1=\text { si el joven pertenece al quintil de ingresos } 5 \\
0=\text { otros casos }\end{array}$ \\
\hline
\end{tabular}

Finalmente, en la tabla 7 se muestran las categorías que fueron consideradas base para la interpretación de los resultados.

Tabla 7. Variables dummy del modelo

\begin{tabular}{|c|c|}
\hline Variable_base & Descripción \\
\hline Rural_5000 & $\begin{array}{l}1=\text { si el joven vive en el área rural } \\
0=\text { otros casos }\end{array}$ \\
\hline Mujer & $\begin{array}{l}1=\text { si el joven es mujer } \\
0=\text { otros casos }\end{array}$ \\
\hline Soltero & $\begin{array}{l}1=\text { si el joven es soltero } \\
0=\text { otros casos }\end{array}$ \\
\hline No_discapacidad & $\begin{array}{l}1=\text { si el joven no tiene alguna discapacidad } \\
0=\text { otros casos }\end{array}$ \\
\hline Mestizo & $\begin{array}{l}1=\text { si el joven es mestizo } \\
0=\text { otros casos }\end{array}$ \\
\hline Madre_no_hogar & $\begin{array}{l}1=\text { si la madre joven no vive en el hogar } \\
0=\text { otros casos }\end{array}$ \\
\hline Ninguna & $\begin{array}{l}1=\text { si la madre joven no tiene ningún tipo de educación } \\
0=\text { otros casos }\end{array}$ \\
\hline Quintil_1 & $\begin{array}{l}1=\text { si el joven pertenece al quintil de ingresos } 1 \\
0=\text { caso contrario }\end{array}$ \\
\hline
\end{tabular}

En lo que respecta a la estimación que considera variable dependiente a la intensidad de la condición nini, se estima un modelo logit ordenado con una variable dependiente que toma el valor de 1 si el joven presenta las características de un desempleado convencional, 2 si el joven está desempleado, 
no estudia pero tiene ganas de trabajar, y 3 si el joven tiene la categoría de desenganchado.

Las estimaciones fueron realizadas ponderadas ya que las variables área y sexo, en comparación con las proyecciones censales, estaban sobrerrepresentadas. Solon, Haider y Wooldridge (2015) justifican que se realice este procedimiento para evitar problemas en la estimación.

\section{Resultados}

La tabla 7 contiene los resultados de la estimación. El modelo 1 (M1) contiene variables innatas de los jóvenes como la edad, el sexo y su etnia. En el modelo 2 (M2), además de las variables del M1, se incluyen aquellas características que se van adquiriendo a lo largo de la vida, como el área en la que reside en la actualidad, el estado civil y la posibilidad de que la madre viva o no en el hogar. Al modelo 3 (M3) se añaden las variables que corresponden a la discapacidad y al ingreso per cápita, el cual se encuentra agrupado por quintiles. Al modelo completo, modelo 4 (M4) se suman las variables relacionadas con el nivel de escolaridad del joven.

Tabla 7. Modelos logit para los ninis ecuatorianos

\begin{tabular}{lcccc}
\hline \multirow{2}{*}{ Variables } & Modelo 1 & Modelo 2 & Modelo 3 & Modelo 4 \\
\cline { 2 - 5 } Edad & nini & nini & nini & nini \\
\hline \multirow{2}{*}{ Edad_cuadrado } & $\left(0,029^{* * *}\right.$ & $2,024^{* * *}$ & $2,098^{* * *}$ & $2,192^{* * *}$ \\
& $-0,0478^{* * *}$ & $-0,0493^{* * *}$ & $-0,0507^{* * *}$ & $-0,0521^{* * *}$ \\
\hline \multirow{2}{*}{ Hombre } & $(0,000249)$ & $(0,000251)$ & $(0,000254)$ & $(0,000262)$ \\
\hline \multirow{2}{*}{ Indígena } & $-1,236^{* * *}$ & $-1,097^{* * *}$ & $-1,142^{* * *}$ & $-1,232^{* * *}$ \\
& $(0,00365)$ & $(0,00369)$ & $(0,00379)$ & $(0,00388)$ \\
\hline \multirow{2}{*}{ Afrodescendiente } & $-0,526^{* * *}$ & $-0,515^{* * *}$ & $-0,620^{* * *}$ & $-0,689^{* * * *}$ \\
& $(0,00739)$ & $(0,00785)$ & $(0,00791)$ & $(0,00809)$ \\
\hline Mlanco & $0,514^{* * *}$ & $0,470^{* * *}$ & $0,348^{* * *}$ & $0,291^{* * *}$ \\
\hline & $(0,00670)$ & $(0,00701)$ & $(0,00723)$ & $(0,00731)$ \\
\hline
\end{tabular}




\begin{tabular}{|c|c|c|c|c|}
\hline \multirow{2}{*}{ Variables } & Modelo 1 & Modelo 2 & Modelo 3 & Modelo 4 \\
\hline & nini & nini & nini & nini \\
\hline \multirow{2}{*}{ Urbano_5000 } & & $0,201^{* * *}$ & $0,338^{* * *}$ & $0,416^{* * *}$ \\
\hline & & $(0,00387)$ & $(0,00402)$ & $(0,00412)$ \\
\hline \multirow{2}{*}{ Casado } & & $0,885^{* * *}$ & $0,897^{* * *}$ & $0,807^{* * *}$ \\
\hline & & $(0,00482)$ & $(0,00498)$ & $(0,00513)$ \\
\hline \multirow{2}{*}{ Separado } & & $0,536^{* * *}$ & $0,412^{* * *}$ & $0,281^{* * *}$ \\
\hline & & $(0,00847)$ & $(0,00872)$ & $(0,00904)$ \\
\hline \multirow{2}{*}{ Madre_hogar } & & $-0,101^{* * *}$ & $-0,0691^{* * *}$ & $-0,00507$ \\
\hline & & $(0,00453)$ & $(0,00467)$ & $(0,00478)$ \\
\hline \multirow{2}{*}{ Discapacidad } & & & $1,833^{* * *}$ & $1,372^{* * *}$ \\
\hline & & & $(0,0102)$ & $(0,0114)$ \\
\hline \multirow{2}{*}{ Quintil_2 } & & & $-0,228^{* * *}$ & $-0,229^{* * *}$ \\
\hline & & & $(0,00545)$ & $(0,00553)$ \\
\hline \multirow{2}{*}{ Quintil_3 } & & & $-0,371^{* * *}$ & $-0,341^{* * *}$ \\
\hline & & & $(0,00537)$ & $(0,00545)$ \\
\hline \multirow{2}{*}{ Quintil_4 } & & & $-0,622^{* * *}$ & $-0,587^{* * *}$ \\
\hline & & & $(0,00549)$ & $(0,00561)$ \\
\hline \multirow{2}{*}{ Quintil_5 } & & & $-1,027^{* * *}$ & $-0,863^{* * *}$ \\
\hline & & & $(0,00611)$ & $(0,00621)$ \\
\hline \multirow{2}{*}{ Alfabetización } & & & & $-2,256^{* * *}$ \\
\hline & & & & $(0,0582)$ \\
\hline \multirow{2}{*}{ Básica } & & & & $-1,913^{* * *}$ \\
\hline & & & & $(0,0204)$ \\
\hline \multirow{2}{*}{ Secundaria } & & & & $-1,911^{* * *}$ \\
\hline & & & & $(0,0205)$ \\
\hline \multirow{2}{*}{ Superior } & & & & $-2,976^{* * *}$ \\
\hline & & & & $(0,0214)$ \\
\hline \multirow{2}{*}{ Constante } & $-22,05^{* * *}$ & $-21,75^{* * *}$ & $-22,33^{* * *}$ & $-21,68^{* * *}$ \\
\hline & $(0,0954)$ & $(0,0961)$ & $(0,0978)$ & $(0,103)$ \\
\hline Observaciones & 2.577.347 & 2.577.347 & 2.577 .347 & 2.577 .347 \\
\hline AIC & 2243869 & 2185584 & 2113886 & 2072700 \\
\hline $\mathrm{R} 2$ & 0,813 & 0,818 & 0,828 & 0,835 \\
\hline Cociente de verosimilitud & 0,099 & 0,122 & 0,151 & 0,168 \\
\hline
\end{tabular}

AIC: criterio de información de Akaike. 
Con el objetivo de realizar una interpretación en términos de la probabilidad de ocurrencia de pertenecer al grupo nini, en la tabla 8 se presenta el efecto marginal del modelo completo y la interpretación se realizará con estos resultados.

Tabla 8. Probabilidad de ocurrencia de las variables del modelo 4

\begin{tabular}{|c|c|c|}
\hline & Variable & Modelo 4 nini \\
\hline 15 a 24 años & Edad & 0,4445 \\
\hline Urbano_5000 & \multirow{2}{*}{ Área } & 0,2053 \\
\hline Rural_5000 & & 0,1547 \\
\hline Hombre & \multirow{2}{*}{ Sexo } & 0,1080 \\
\hline Mujer & & 0,2637 \\
\hline Soltero & \multirow{3}{*}{ Estado civil } & 0,1539 \\
\hline Casado & & $0,26 \mathrm{t} 93$ \\
\hline Separado & & 0,2237 \\
\hline Tiene discapacidad & \multirow{2}{*}{ Discapacidad } & 0,4041 \\
\hline No tiene discapacidad & & 0,1830 \\
\hline Mestizos & \multirow{5}{*}{ Etnia } & 0,1882 \\
\hline Indígena & & 0,1183 \\
\hline Afrodescendiente & & 0,2242 \\
\hline Montuvio & & 0,2271 \\
\hline Blanco & & 0,2180 \\
\hline Está en el hogar & \multirow{2}{*}{ Madre en el hogar } & 0,1875 \\
\hline No está en el hogar & & 0,1882 \\
\hline Alfabetización & \multirow{5}{*}{ Educación del joven } & 0,0307 \\
\hline Educación básica & & 0,0896 \\
\hline Educación secundaria & & 0,1257 \\
\hline Educación superior & & 0,0248 \\
\hline Ninguna & & 0,5416 \\
\hline Quintil 1 & \multirow{5}{*}{ Quintiles } & 0,2458 \\
\hline Quintil 2 & & 0,1659 \\
\hline Quintil 3 & & 0,1567 \\
\hline Quintil 4 & & 0,1377 \\
\hline Quintil 5 & & 0,1134 \\
\hline \multicolumn{3}{|c|}{ Combinaciones entre variables } \\
\hline 15 a 20 años & \multirow{2}{*}{ Rango de edad } & 0,0079 \\
\hline 20 a 24 años & & 0,5382 \\
\hline
\end{tabular}




\begin{tabular}{lcc}
\hline & Variable & Modelo 4 nini \\
\hline Hombre del área urbana & Sexo_área & 0,1204 \\
Mujer del área urbana & & 0,2867 \\
\hline Hombre con discapacidad & Sexo_discapacidad & 0,2817 \\
Mujer con discapacidad & & 0,5267 \\
\hline Hombres casados & Sexo_estado civil & 0,1654 \\
Mujeres casadas & & 0,3740 \\
Hombres solteros & & 0,2237 \\
Mujeres solteras & & 0,1075 \\
\hline Hombre mestizo & & 0,2645 \\
Mujer mestiza & & 0,1336 \\
Hombre afrodescendiente & Sexo_etnia & 0,3111 \\
Mujer afrodescendiente & & 0,1357 \\
Hombre montuvio & 0,3148 \\
Mujer montuvia & & \\
\hline
\end{tabular}

Al realizar un análisis por grupos de edad, se observa que los jóvenes que se encuentran entre los 20 y 24 años son los más propensos a convertirse en ninis. Estos resultados se asemejan con algunos de los estudios realizados para América Latina (Navarrete et al., 2011; Elizondo \& Avellaneda, 2015; Comari, 2015; Bolaños \& Rivera, 2016), los cuales sugieren una mayor concentración de jóvenes ninis a medida que la edad aumenta.

Estos resultados coinciden con la implementación del Sistema Nacional de Nivelación y Admisión — SNNA - en el 2012, que tiene por objetivo incrementar el acceso al sistema de educación superior, sin discriminación a ningún grupo poblacional y basándose en principios de igualdad de oportunidades, transparencia y méritos, y que ha generado un incremento de la tasa bruta de matrícula universitaria en 9,25\% en el periodo que va de 1999 al 2014 (Senescyt, 2015). En la práctica, este nuevo sistema se traduce a que los estudiantes deben rendir una prueba de aptitud abstracta, dominio matemático, lingüístico, científico y social, y la asignación de cupos y carreras es en función del rendimiento en dicha prueba. Sin embargo, es necesario analizar si este incremento benefició a los jóvenes pertenecientes a minorías étnicas, a los quintiles de renta más bajos y, especialmente, analizar las condiciones y el tiempo que transcurre para que un joven pueda ingresar a una universidad.

En cuanto al área, urbana o rural, existe una mayor probabilidad de que el joven nini se encuentre en las zonas urbanas del país. Según el informe de la Corporación Andina de Fomento - CAF- (2016), los jóvenes que habitan en el área rural tienen menos probabilidades de ser ninis, pues a pesar de 
que no puedan asistir a la escuela, tienen posibilidades de trabajar en tareas agrícolas. Adicionalmente, en caso de que el joven sea hombre y viva en el área urbana, la probabilidad de ser nini es del 12,04\%, mientras que para las mujeres es del 28,67\%.

En cuanto a los resultados por sexo, se obtuvo que son las mujeres quienes tienen una mayor probabilidad de convertirse en ninis, con $26,37 \%$, en comparación con los hombres, cuya probabilidad es de 10,80\%, tal y como se muestra en la figura 11.

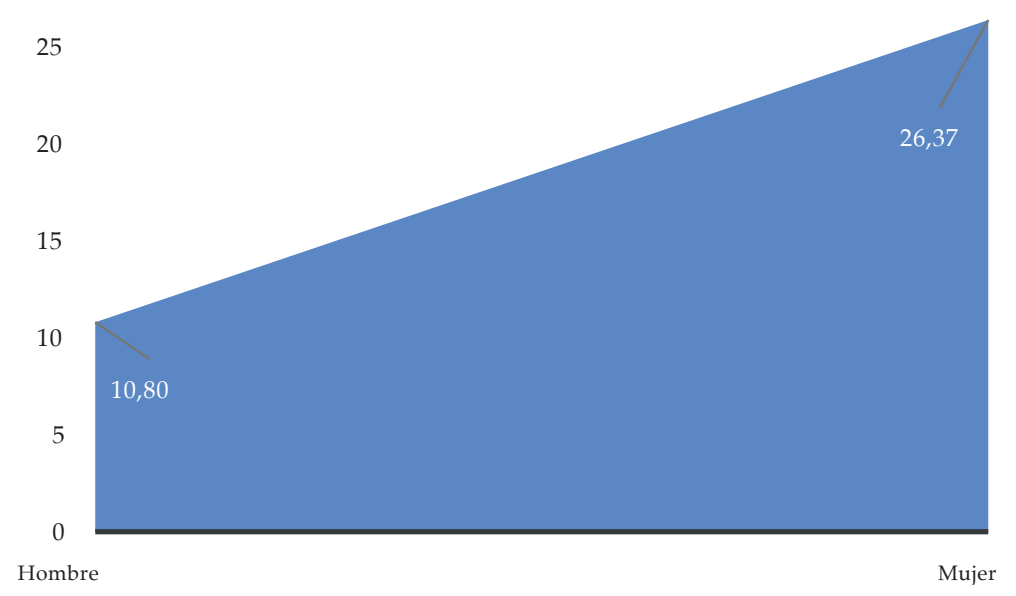

Figura 11. Probabilidad de que el joven se convierta en nini según el sexo

Contrario a la creencia de que son los hombres los más propensos a permanecer en casa sin realizar alguna actividad, se observa que son las mujeres las más propensas a caer en esta situación (Aguayo et al., 2013). Estos resultados también pueden ser comparados con estudios realizados para distintos países de América Latina (Navarrete et al., 2011; Aguayo et al., 2013; Elizondo \& Avellaneda, 2015; Comari, 2015; Bolaños \& Rivera, 2016; Ospina et al., 2017), los cuales indican que la mayor concentración de ninis está presente en las mujeres. En cuanto a este aspecto, la Cepal (2014) menciona que las razones que influyen para que se dé esta desigualdad en las cifras son: el embarazo adolescente para el caso de las mujeres, junto con la tendencia a quedarse en sus hogares para la realización del trabajo doméstico, sumando a esto factores culturales, ya que en ciertos lugares generalmente las hijas mujeres deben ayudar a sus madres en el hogar y en el cuidado de sus hermanos más pequeños. Además, el Banco Mundial (De Hoyos et al., 2016) señala que esto se da debido a que las mujeres siguen viviendo con sus padres y no buscan empleo. Por último, puede darse el caso de que las jóvenes ya han formado 
un nuevo hogar y tienen hijos, pero por su situación de desempleadas deben permanecer en la vivienda de sus padres.

En cuanto al estado civil, se observa que en Ecuador los jóvenes casados o separados tienen mayor probabilidad de convertirse en nini, con un $26,93 \%$ y 22,37\%, respectivamente, en comparación con los jóvenes ninis que son solteros, que tienen una probabilidad de $15,39 \%$. Además, si el joven es hombre y está casado tiene un $16,54 \%$ de posibilidad de ser nini pero, si es mujer la probabilidad es de $37,40 \%$. Por otro lado, si el joven es hombre y está soltero la probabilidad es del $8,51 \%$, mientras que para las mujeres la probabilidad asciende al $22,37 \%$. Como se puede evidenciar, en cuanto al análisis del estado civil por sexo, se sigue teniendo una mayor incidencia en las mujeres para convertirse en ninis, lo cual se debe a que al momento de que las jóvenes forman sus hogares, según Málaga et al. (2014), sus cónyuges se dedican a trabajar y ellas se quedan al cuidado del hogar. El mismo autor menciona que, asociado con este estado civil, los jóvenes al no tener una vida sexual responsable, tienen hijos prematuramente, por lo que deben enfrentar grandes desafíos debido a la mayor responsabilidad que eso significa. En definitiva, el aumento de los ninis para el caso de las mujeres responde al hecho de que tener hijos puede llevarlas a la deserción escolar o simplemente dejar los estudios para conseguir un empleo, y en los varones la responsabilidad de tener hijos los incentivaría a ingresar con brevedad al mercado laboral para generar ingresos. Navarrete et al. (2011) también mencionan que en España existe un mayor porcentaje de jóvenes ninis casados o separados. Así mismo, el Fondo Internacional de Emergencia de las Naciones Unidas para la Infancia (Unicef, 2001) afirma que el matrimonio, inevitablemente, niega a los niños y los jóvenes en edad escolar (en su mayoría menores de 18 años) el derecho a recibir la educación que necesitan para completar su desarrollo personal y su preparación para la edad adulta, por lo que no contribuyen al futuro bienestar de su familia, ni de la sociedad en la que viven.

En relación con la etnia, se obtuvo que cuando el joven es afrodescendiente, montuvio o blanco aumenta la probabilidad de convertirse en nini en un $22,42 \%, 22,71 \%$ y $21,80 \%$, respectivamente, en comparación con los mestizos, cuya probabilidad es del $18,82 \%$ y $11,83 \%$ para los indígenas. Por su parte, si un joven es hombre y afrodescendiente tiene un $13,36 \%$ de posibilidad de ser nini, mientras que si es mujer la probabilidad asciende al 31,11\%. Para el caso de los hombres montuvios, la probabilidad de convertirse en nini es de $13,57 \%$ frente a un $31,48 \%$ para el caso de las mujeres; estos resultados son mayores comparando con los mestizos, pues se observa un 10,75\% para los hombres y un $26,45 \%$ para las mujeres. Al igual que en el caso geográfico, en cuanto a etnia, no existe suficiente información acerca de qué porcentaje de 
ninis está dentro de cada grupo étnico, puesto que para cada país es diferente la constitución de estos grupos, resultando de esto una difícil caracterización de jóvenes ninis por etnia a nivel de regiones. Además, la mayoría de estudios que mencionan a las minorías étnicas representan a toda la población, mas no a un grupo etario específico.

Tomando el tema de la discapacidad como factor determinante de que los jóvenes se conviertan o no en ninis en Ecuador, se tuvo como resultado que si la persona es discapacitada la posibilidad de ser nini es del 40,41\%. Analizando este factor por sexo, si el joven es hombre la probabilidad de que se convierta en nini es del $28,17 \%$ y para una mujer con discapacidad asciende al 52,67\%. De la misma manera, autores como Málaga et al. (2014) y Comari (2015) mencionan que existe una correlación positiva entre la existencia de jóvenes con discapacidad y jóvenes que se convierten en nini, además de que esta característica se acentúa más cuando el tipo de discapacidad es fuerte o total. Como se conoce, la condición de discapacidad no depende de la persona, ya que puede ser congénita o haberla adquirido con el paso del tiempo. Por tanto, no depende de la voluntad de este grupo de jóvenes convertirse en ninis, sino más bien de sus circunstancias, ya que dependiendo del nivel de su discapacidad se ven imposibilitados para el trabajo y para estudiar. En este último caso, se debe tener en cuenta que ciertos centros educativos no cuentan con la infraestructura adecuada para que jóvenes discapacitados puedan asistir a clases con normalidad.

Continuando con el análisis del factor estructura familiar, los resultados del modelo para Ecuador explican que el hecho de que los padres vivan en el hogar disminuye la probabilidad de que el joven se convierta en nini. Específicamente, se observa que en el caso de que la madre viva en el hogar con su hijo la probabilidad de que se convierta en nini disminuye en un 18,75\%. Román (2013) expresa que el tipo de estructura y organización familiar es un factor que dificulta la trayectoria escolar del joven, o, a su vez, es desencadenante tanto del retiro como del abandono del sistema. Además, Mahmoudian y Torkashvand (2013) afirman que el papel de la familia dentro del grupo de jóvenes de 15 a 19 años es fundamental, pues son responsables de todas las oportunidades y las limitaciones relacionadas con la consecución de capital humano, ya que la ausencia de un padre puede tener un impacto en la cantidad de recursos disponibles para los miembros de la familia.

Referente a la educación del joven, los resultados que se obtuvieron en el modelo muestran que la probabilidad de convertirse en nini disminuye mayormente cuando el joven ha cursado la educación secundaria, con un $12,57 \%$, mientras que la probabilidad de convertirse en nini si fue a algún centro de alfabetización o cursó la educación básica es de 3,07\% y 8,96\%, 
respectivamente. Un joven que no tiene ningún tipo de educación tiene una probabilidad de convertirse en nini del 54,16\%.

Para los ingresos, se obtuvo que la renta percibida por un hogar también desempeña un papel decisivo para que el joven se convierta o no en nini. El modelo sugiere que mientras mayor es el ingreso per cápita de las personas dentro del hogar, menor es la probabilidad de que el joven sea nini. Así, para los quintiles 1, 2, 3, 4 y 5 las probabilidades son del 24,58 \%, 16,59\%, 15,67\%, $13,77 \%$ y $11,34 \%$, respectivamente (figura 12). Siguiendo con esta tendencia, se observa que los ninis se encuentran principalmente en hogares que pertenecen a los quintiles más bajos o estratos medio-bajo de ingreso (Monteiro, 2013; Elizondo y Avellaneda, 2015; Bolaños y Rivera, 2016).

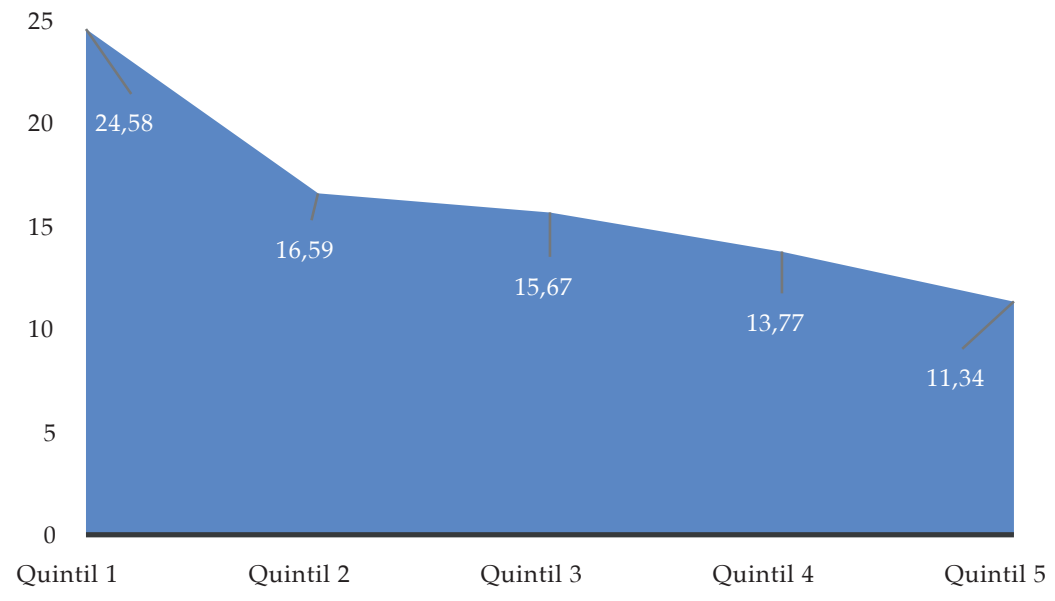

Figura 13. Probabilidad de que el joven se convierta en nini según quintiles de ingresos, 2014

Con lo expuesto, es preciso realizar un análisis de manera conjunta en cuanto a los ingresos y la educación de la persona, puesto que existe una relación positiva entre estas variables, ya que una mayor educación produce mayores ingresos para el individuo (Becker, 1964; Mincer, 1974; Stiglitz, 1975). Estos resultados podrían reflejar que los jóvenes dejan de estudiar en gran parte por la pobreza, pues no van a la escuela porque no tienen suficiente dinero para pagar los costos o porque necesitan trabajar para ayudar a sus familias.

Becker (2013) también afirma que los ingresos salariales a lo largo de la vida de los hijos tienen una tendencia creciente en promedio del $4 \%$ cuando los ingresos de los padres aumentan en un $10 \%$, por tanto, el grado de movilidad intergeneracional aumentaría, es decir, el hecho de que los padres tengan ingresos altos contribuye a que los hijos tengan en un futuro ingresos 
altos, disminuyendo la brecha intergeneracional, en la que cada vez existirán menos personas que dejan de estudiar por falta de recursos. También, surgen otras consideraciones que deben ser tomadas en cuenta, como lo mencionan Pérez y Romero (2012), pues los hogares de bajos ingresos procuran tener mayores entradas, por lo que los jóvenes que pertenecen a estos hogares tienen que salir a emplearse.

Dado que la variable relacionada con el nivel de educación de los padres presenta un alto porcentaje de datos perdidos (68\% y $50 \%$ para madres y padres, respectivamente), no se colocó en los modelos principales. Sin embargo, se estimó un modelo eliminando las observaciones de no respuesta para evidenciar cómo influye esta variable en que el joven se convierta o no en nini. En la estimación realizada se pudo reflejar que el nivel educativo de los padres es un factor determinante que incide en la formación de los jóvenes ninis, ya que si los padres tienen un menor nivel de educación, la probabilidad aumenta, pues no verían en la educación un mecanismo que otorga beneficios a futuro, existiendo así altas tasas de deserción escolar y de jóvenes desempeñándose en empleos informales. Además, los padres al no tener un nivel alto de educación no pueden desempeñarse en trabajos donde reciban un salario que permita mantener el hogar y deben recurrir a enviar a sus hijos a trabajar para ayudar con la solvencia de los gastos.

En la tabla 8 se presenta la estimación que toma en consideración la intensidad de la condición nini. Únicamente se mencionarán las variables que obtuvieron un signo contrario con respecto a los resultados obtenidos en la tabla 7 .

Tabla 8. Modelo logit ordenado-intensidad de la condición nini

\begin{tabular}{lc}
\hline Variables & Modelo 5 nini \\
\hline Edad & $-1,3948^{* * *}$ \\
& $(0,0610)$ \\
\hline Edad_cuadrado & $0,0302^{* * *}$ \\
\hline Urbano_5000 & $(0,0015)$ \\
\hline Hombre & $0,1636^{* * *}$ \\
& $(0,0151)$ \\
\hline Indígena & $-3,0082^{* * *}$ \\
\hline
\end{tabular}




\begin{tabular}{|c|c|}
\hline Variables & Modelo 5 nini \\
\hline \multirow{2}{*}{ Afrodescendiente } & $-0,3500^{* * *}$ \\
\hline & $(0,0260)$ \\
\hline \multirow{2}{*}{ Montuvio } & $0,2118^{* * *}$ \\
\hline & $(0,0240)$ \\
\hline \multirow{2}{*}{ Blanco } & $-0,0243$ \\
\hline & $(0,0407)$ \\
\hline \multirow{2}{*}{ Casado } & $0,6536^{* * *}$ \\
\hline & $(0,0256)$ \\
\hline \multirow{2}{*}{ Separado } & $-0,9161^{* * *}$ \\
\hline & $(0,0381)$ \\
\hline \multirow{2}{*}{ Discapacidad } & \\
\hline & $(0,0626)$ \\
\hline \multirow{2}{*}{ Quintil_2 } & $0,0995^{* * *}$ \\
\hline & $(0,0215)$ \\
\hline \multirow{2}{*}{ Quintil_3 } & $-0,1350^{* * *}$ \\
\hline & $(0,0182)$ \\
\hline \multirow{2}{*}{ Quintil_4 } & $-0,1524^{* * *}$ \\
\hline & $(0,0210)$ \\
\hline \multirow{2}{*}{ Quintil_5 } & $-0,0979^{* * *}$ \\
\hline & $(0,0258)$ \\
\hline \multicolumn{2}{|c|}{ Corte 1} \\
\hline \multirow{2}{*}{ Constante } & $-21,4426^{* * *}$ \\
\hline & $(0,6381)$ \\
\hline \multicolumn{2}{|l|}{ Corte 2} \\
\hline \multirow{2}{*}{ Constante } & $-17,3685^{* * *}$ \\
\hline & $(0,6310)$ \\
\hline $\mathrm{N}$ & 218022 \\
\hline AIC & 165013,3 \\
\hline Pseudo $\mathrm{R}^{2}$ & 165188,3 \\
\hline
\end{tabular}

La edad es una variable significativa con signo negativo, lo que sugiere que a medida que los jóvenes aumentan su edad disminuye la intensidad de la inactividad; lo mismo sucede con los jóvenes ninis de sexo masculino. 
Estos resultados podrían interpretarse como un reflejo de que a medida que pasa el tiempo, los jóvenes, y especialmente los hombres, pueden motivarse para salir del estado de desvinculados.

De esta manera, se ha logrado obtener un panorama general sobre la población de jóvenes ninis en el Ecuador, quienes ni estudian ni trabajan, identificando características tanto individuales como del hogar.

\section{Conclusiones y recomendaciones}

Dentro de la población de jóvenes ecuatorianos con edades comprendidas entre 15 y 24 años, el 19\% no asiste a ningún centro educativo y está desempleado. Estos jóvenes son los denominados ninis y las consecuencias de su inactividad laboral y educativa tienen afectación a nivel individual y para toda la sociedad, pues son individuos improductivos y poco calificados, y su condición podría favorecer al incremento de la pobreza y la desigualdad. Como resultado de la investigación, se pudo determinar que, en el Ecuador, la población de jóvenes entre 15 y 24 años va en aumento en términos absolutos año a año. El análisis realizado refleja que la mayoría de los ninis son mujeres, pertenecientes a los quintiles de ingreso más bajos, y que la mayor incidencia de ninis se presenta en los afrodescendientes y montuvios y están localizados en las áreas urbanas. Así, los resultados sugieren que las políticas encaminadas a controlar el crecimiento de los jóvenes ninis deben una vez más orientarse a los grupos vulnerables del Ecuador. Si bien la implementación del nuevo sistema de ingreso a las universidades tiene como objetivo garantizar el acceso a la universidad en términos del mérito individual, es necesario revisar si los pertenecientes a grupos en desventaja socioeconómica están aptos para competir o si ante las circunstancias de desigualdad su opción es abandonar la carrera y convertirse en nini. Adicionalmente, el solo ingreso a la universidad no garantiza la permanencia de los estudiantes en esta. La concesión de becas al mérito y por situación económica debe continuar como una política prioritaria de las universidades del país. El crecimiento de ninis en el Ecuador también puede obedecer a que la tasa de desempleo en el rango de 15 a 24 años ha ido aumentando. El gobierno ecuatoriano ha impulsado algunas políticas de empleo juvenil, como Mi Primer Empleo y la Ley Orgánica para la Promoción del Empleo Juvenil, sin embargo, la estabilidad y el crecimiento en la cifra de los ninis ecuatorianos sugiere un análisis de estos temas con el objetivo de evaluar su efectividad. 


\section{Referencias}

Acevedo, M., Montes, I., Vásquez, J., Villegas, M. \& Brito, T. (2007). Capital humano: una mirada desde la educación y la experiencia laboral. Cuadernos de Investigación, (56), 1-40.

Aguayo, E., Mancha Torres, G. L. \& Rangel González, E. (2013). Descifrando a los ninis: un estudio para Nuevo León y México. Monterrey: Universidad Autónoma de Nuevo León.

Almeida, R., Fitzsimons, E. \& Rogers, H. (2015). How to prevent secondaryschool dropout: evidence from rigorous evaluations. Washington, D.C.: Banco Mundial.

Angrist, J. \& Lavy, V. (2009). The effects of high stakes high school achievement awards: evidence from a randomized trial. American Economic Review, 99(4), 1384-1414.

Barr, N. (2012). Economics of the welfare state ( $5^{\mathrm{a}}$ ed.). Gosport: Oxford University Press.

Becker, G. (1964). Human capital. Nueva York: The National Bureau of Economic Research.

Becker, G. S. (1962). Investment in human capital: a theoretical analysis. The Journal of Political Economy, 70(5), 9-49.

Becker, G. S. (2002). The age of human capital. Recuperado de http:/ / media. hoover.org/sites/default/files/documents/0817928928_3.pdf

Becker, G. (1 de enero del 2013). Meritocracies and intergeneration mobility. The Becker-Posner Blog. Recuperado de http: / / www.becker-posner-blog .com/2013/01/meritocracies-and-intergeneration -mobility-becker.html

Bell, L. \& Thurlby, I. (2017). Agency, structure and the NEET policy problem: the experiences of young people. Nueva York: Bloomsbury Academic.

Berniell, L., De la Mata, D., Bernal, R., Camacho, A., Barrera-Osorio, F., Álvarez, F., Brassiolo, P. \& Vargas, J. F. (2016). Red 2016. Más habilidades para el trabajo y la vida: los aportes de la familia, la escuela, el entorno y el mundo laboral. Bogotá: CAF. Recuperado de http://scioteca.caf. com/handle/123456789/936

Bolaños, F. \& Rivera, M. E. (2016). Los jóvenes nini en El Salvador. El Salvador: Fundaungo. Recuperado de http:/ /www.fundaungo.org.sv/www/ pdf/2016/Los_j\%C3\%B3venes_nini_en_El_Salvador.pdf

Brada, J., Marelli, E. \& Signorelli, M. (2014). Introduction: young people and the labor market: key determinants and new evidence. Comparative Economic Studies, 56(4), 556-566. 
Chiapa, C. \& Juarez, L. (2012). The schooling repayment hypothesis for private transfers: evidence from the Progresa/Oportunidades experiment. Review of Economics of the Household, 14(4) 811-828.

Comari, C. (2015). Examen de validez teórica y empírica del concepto "jóvenes nini" o "generación nini" en la Argentina del Siglo xxI (tesis inédita de doctorado). Instituto Nacional de Estadística y Censos -Indec-, Ciudad Autónoma de Buenos Aires, Argentina.

Comisión Económica para América Latina y el Caribe -Cepal- (2014). Panorama social de América Latina. Santiago de Chile: United Nations Publications.

Craig, L., Powell, A. \& Brown, J. E. (2015). Co-resident parents and young people aged 15-34: who does what housework? Social Indicators Research, 121(2), 569-588.

Currie, J. \& Almond, D. (2011). Human capital development before age five. Handbook of Labor Economics, 4, 1315-1486.

De Hoyos, R., Rogers, H. \& Székely, M. (2016). Ninis en América Latina: 20 millones de jóvenes en búsqueda de oportunidades. Washington D.C.: Grupo Banco Mundial.

Elder, S. (2015). What does NEETs mean and why is the concept so easily misinterpreted? Technical Brief n. ${ }^{\circ}$ 1. International Labour Office. Recuperado de http://www.ilo.org/wcmsp5/groups/public/@dgreports/@ dcomm/documents/publication/wcms_343153.pdf

Elizondo, N. A. \& Avellaneda, D. L. (2015). El fenómeno de los jóvenes nini en Chile. Ponencia presentada en el Sexto Encuentro Anual, Chile. Sociedad Chilena de Políticas Públicas. Recuperado de http:/ / www.ced.cl/ced/ wp-content/uploads/2015/01/achpp-ponencia-avellaneda-elizondo.pdf Empreende Ja (2017). Portal da Juventude. Recuperado de https: / juventude. gov.pt/Emprego/Empreende-Ja

Ferreira, F. G., Messina, J., Rigolini, J., López-Calva, L. F. \& Vakis, R. (2013). La movilidad económica y el crecimiento de la clase media en América Latina. Washington, D.C.: Banco Mundial.

Fondo de las Naciones Unidas para la Infancia -Unicef- (2001). Matrimonios prematuros. Diggest Innocenti, 7.

Fundación Europea para la Mejora de las Condiciones de Vida y de Trabajo -Eurofound-(2012). NEETS - Young people not in employment, education or training: characteristics, costs and policy responses in Europe. Luxembourg: Publications Office of the European Union.

Guryan, J., Hurst, E. \& Kearney, M. (2008). Parental education and parental time with children. The Journal of Economic Perspectives, 22(3), 23. 
Hanushek, E. A. \& Woessmann, L. (2008). The role of cognitive skills in economic development. Journal of Economic Literature, 46(3), 607-668.

Heller, S., Pollack, H. A., Ander, R. \& Ludwig, J. (2013). Preventing youth violence and dropout: a randomized field experiment. Informe Técnico. Cambridge: National Bureau of Economic Research.

Instituto Nacional de Estadísticas y Censos - INEC - (1999). Encuesta de Condiciones de Vida. Quito, Ecuador.

Instituto Nacional de Estadísticas y Censos - INEC- (2006). Encuesta de Condiciones de Vida. Quito, Ecuador.

Instituto Nacional de Estadísticas y Censos - INEC- (2014). Encuesta de Condiciones de Vida (ECV). Bases de Datos. Quito, Ecuador. Recuperado de http://www.ecuadorencifras.gob.ec/encuesta-de-condiciones-devida-ecv /

International Labour Office - ILO- (2017). Policy brief on outreach strategies for young NEETS. Informe Técnico. Geneva. Recuperado de http:/ / www. ilo.org/wcmsp5/groups/public/---ed_emp/documents/publication/ wcms_544350.pdf

Istance, D., Rees, G. \& Williamson, H. (1994). Young people not in education, training or employment in South Glamorgan. Glamorgan: South Glamorgan Training and Enterprise Council Cardiff.

Jensen, R. (2010). The (perceived) returns to education and the demand for schooling. Quarterly Journal of Economics, 125(2), 515-548.

Kautz, T., Heckman, J. J., Diris, R., ter Weel, B. \& Borghans, L. (2014). Fostering and measuring skills: improving cognitive and non-cognitive skills to promote lifetime success. París: Secretary-General of the oecD. Recuperado de http:/ / www.oecd.org/education/ceri/Fostering-and-Measuring-SkillsImproving-Cognitive-and-Non-Cognitive-Skills-to-Promote-LifetimeSuccess.pdf

Kosugi, R. (2006). Youth employment in Japan's economic recovery: 'freeters' and 'NEETs'. The Asia-Pacific Journal, 4(5), 1-3.

Maguire, S. (2013). What measures can be taken to address the specific problem of young people who are NEET? Intereconomics. Leibniz Information Centre for Economics.

Mahmoudian, H. \& Torkashvand, M. (2013). Living in single-parent families and the human capital achievement by children aged 15-19 years in urban areas of Iran. Ponencia presentada en la XXVII IUSSP International Population Conference, Busan, Republic of Korea, 26 August-31 August.

Málaga, R., Oré, T. \& Tavera, J. (2014). Jóvenes que no trabajan ni estudian: el caso peruano. Revista Economía, 37(74), 95-132. 
Marshall, T. H. (1950). Citizenship and social class and other essays. Londres: Cambridge University Press/Bentley House, N. W. I.

Mascherini, M. (2017). Good practices in dealing with young people who are NEETS: policy responses at European level. Ponencia presentada en la 2017 Plenary Session Towards a Participatory Society: New Roads to Social and Cultural Integration 28 April-2 May. P. Donati (editor). Acta 21, Vatican City.

Mincer, J. (1958). Investment in human capital and personal income distribution. The Journal of Political Economy, 66(4), 281-302.

Mincer, J. A. (1974). Schooling, experience, and earnings. Human Behavior $\mathcal{E}$ Social Institutions, (2), 41-63.

Martínez, J. (2007). Regímenes del bienestar en América Latina. Documentos de Trabajo (Fundación Carolina), 1(11), 1.

Monteiro, J. (2013). Quem são os jovens nem-nem?: uma análise sobre os jovens que não estudam e não participam do mercado de trabalho. Texto de discussão $n .^{\circ}$ 34. Instituto Brasileiro de Economia. Rio de Janeiro: FGV-IBRE.

Navarrete Moreno, L., Caro Sagüés, G., Carreras, E., de Francisco, R., Gastón Faci, D., Roldán, A., Gutierrez Villalta, J. \& Zúñiga Contreras, R. (2011). Desmontando a ni-ni. un estereotipo juvenil en tiempos de crisis. Instituto de la Juventud de España. Recuperado de http:/ / eprints.ucm. es/33319/1/9206-01.pdf

Negrete, R. \& Leyva, G. (2013). Los NiNis en México: una aproximación crítica a su medición. Revista Internacional de Estadística y Geografía, 4(1), 90-121.

O’Dea, B., Glozier, N., Purcell, R., McGorry, P. D., Scott, J., Feilds, K.-L., Hermens, D. F., Buchanan, J., Scott, E. M., Yung, A. R., Killacky, E., Guastella, A. \& Hickie, I. (2014). A cross-sectional exploration of the clinical characteristics of disengaged (NEET) young people in primary mental healthcare. BMJ Open, 4(12), 1-8. DoI: 10.1136/bmjopen-2014-006378

Organización Internacional del Trabajo - OIT- (2012). La crisis del empleo de los jóvenes: ¡Actuemos ya! Ponencia presentada en la Conferencia Internacional del Trabajo, 101. a reunión, número 5, p. 128, Ginebra. Recuperado de http:/ / www.ilo.org/wcmsp5/groups/public/---ed_norm/-relconf/ documents/meetingdocument/wcms_176940.pdf

Organización de las Naciones Unidas - ONU- (2015). Transformar nuestro mundo: la Agenda 2030 para el Desarrollo Sostenible 2015. Recuperado de http:/ / www.un.org/ga/search/view_doc.asp?symbol=A/ RES/70/1\&referer=http: / / www.un.org/sustainabledevelopment / sustainable-development-goals / \&Lang=S

Ospina, V., Cartagena, A., García-Suaza, J., Guataquí, I. \& Jaramillo, J. (2017). Informe 2: perfil juvenil urbano de la inactividad y el desempleo en Colombia. Bogotá: Observatorio Laboral de la Universidad del Rosario (LaboUR). 
Pérez Sosto, G. \& Romero, M. (2012). Futuros inciertos. Informe sobre vulnerabilidad, precariedad y desafiliación de los jóvenes en el conurbano bonaerense. Buenos Aires: Unesco.

Ramírez, G. \& Corvo, M. (2007). Causas de deserción de alumnos de primeros semestresde una universidad privada. Revista Mexicana de Orientación Educativa, 5(12), 34-39.

Rodríguez, A. A. \& Vindas, M. A. S. (2011). La deserción estudiantil en la educación superior: el caso de la Universidad de Costa Rica. Revista Actualidades Investigativas en Educación, 5(4), 1-22.

Román, M. (2013). Factores asociados al abandono y la deserción escolar en América Latina: una mirada de conjunto. Reice. Revista Electrónica Iberoamericana sobre Calidad, Eficacia y Cambio en Educación, 11(2), 33-59.

Schultz, T. W. (1961). Investment in human capital. The American Economic Review, 51(1), 1-17.

Secretaría de Educación Superior, Ciencia, Tecnología e Innovación -Senescyt - . (2015). Informe rendición de cuentas año fiscal 2015, Quito Ecuador Recuperado de http:/ / www.senescyt.gob.ec/rendicion2015/ assets/informe-de-rendici\%C3\%B3n-de-cuentas-2015.pdf

Serracant, P. (2014). A brute indicator for a NEET case: genesis and evolution of a problematic concept and results from an alternative indicator. Social Indicators Research, 117(2), 401-419.

Smith, A. (1952). An inquiry into the nature and causes of the wealth of nations. En Great books of the Western world. Vol. 39. Chicago, Encyclopedia Britannica (1955). Recuperado de http:/ /gen.lib.rus.ec/book/index.ph p?md5=1fb233a7ad96ad92bf47e24344837457

Solon, G., Haider, S. J. \& Wooldridge, J. M. (2015). What are we weighting for? Journal of Human Resources, 50(2), 301-316.

Stiglitz, J. (1975). The theory of screening, education, and the distribution of income. American Economic Review, 65(3), 283-300.

Vakis, R., Rigolini, J. \& Lucchetti, L. (2015). Left behind: chronic poverty in Latin America and the Caribbean. Washington, D.C.: World Bank Publications. 